\title{
Logarithmic terms in the soft expansion in four dimensions
}

\author{
Alok Laddha ${ }^{a}$ and Ashoke Sen ${ }^{b}$ \\ ${ }^{a}$ Chennai Mathematical Institute, \\ SIPCOT IT Park, Siruseri, Chennai, India \\ ${ }^{b}$ Harish-Chandra Research Institute, HBNI, \\ Chhatnag Road, Jhusi, Allahabad 211019, India \\ E-mail: aladdha@cmi.ac.in, sen@hri.res.in
}

\begin{abstract}
It has been shown that in larger than four space-time dimensions, soft factors that relate the amplitudes with a soft photon or graviton to amplitudes without the soft particle also determine the low frequency radiative part of the electromagnetic and gravitational fields during classical scattering. In four dimensions the S-matrix becomes infrared divergent making the usual definition of the soft factor ambiguous beyond the leading order. However the radiative parts of the electromagnetic and gravitational fields provide an unambiguous definition of soft factor in the classical limit up to the usual gauge ambiguity. We show that the soft factor defined this way develops terms involving logarithm of the energy of the soft particle at the subleading order in the soft expansion.
\end{abstract}

Keywords: Classical Theories of Gravity, Scattering Amplitudes

ARXIV EPRINT: 1804.09193 


\section{Contents}

1 Introduction and summary 1

2 Logarithmic corrections from soft factors 5

$\begin{array}{llr}3 & \text { Some relevant integrals } & 8\end{array}$

4 Electromagnetic radiation $\quad 10$

5 Gravitational radiation $\quad 11$

5.1 Gravitational radiation from scattering via electromagnetic interaction 11

$\begin{array}{lll}5.2 & \text { Gravitational radiation from scattering via gravitational interaction } & 15\end{array}$

$\begin{array}{ll}\text { A Evaluation of some integrals } & \mathbf{2 0}\end{array}$

A.1 Evaluation of $I_{1} \quad 21$

A.2 Evaluation of $I_{2} \quad 22$

A.3 Evaluation of $I_{3} \equiv \int_{-\infty}^{\infty} d t(r(t))^{-1} f(t)\left[e^{-i \omega g(t)}-e^{-i \omega h(t)}\right] \quad 22$

A.4 Evaluation of $I_{4} \equiv \omega^{-1} \int_{-\infty}^{\infty} d t(r(t))^{-2} f(t)\left[e^{-i \omega g(t)}-e^{-i \omega h(t)}\right] \quad 23$

A.5 Evaluation of $I_{5} \equiv \int_{-\infty}^{\infty} d t(r(t))^{-1} f(t) e^{-i \omega g(t)}$

\section{Introduction and summary}

Soft theorems, that relate an amplitude with soft photons or gravitons to amplitudes without any soft particle [1-12], have been investigated intensively in recent years [13-60], partly due to their connection to asymptotic symmetries [61-84]. Much of the discussion that relates soft theorem to asymptotic symmetries has been in the context of four dimensional theories, although there are some exceptions. However in four space-time dimensions the S-matrix suffers from infrared divergences and is ill-defined. Therefore it is not obvious what soft theorem means in four space-time dimensions beyond tree level. Indeed, in the case of gravity and abelian gauge theory, it has been shown that the leading soft factors are universal and are insensitive to infrared loop effects $[2,8]$, but the subleading soft factors are infra-red divergent and can only be defined with appropriate regularization schemes [17, 20, 75]. For Yang-Mills theory, even the leading order soft factor is not universal once loop effects are taken into account and becomes regularization dependent [17].

In [85] it was shown that in generic space-time dimensions, by taking classical limit of multiple soft theorem, where we take the energies / charges of the finite energy external states to be large, one can relate soft factors to the power spectrum of low frequency classical radiation during a scattering process. Since the latter can also be expressed in terms of the radiative components of the electromagnetic and gravitational fields, this analysis yields a relation between the soft factors and the radiative components of low frequency electromagnetic and gravitational fields. If we take this relationship between 
classical radiation and soft theorem as the definition of the classical soft factor, ${ }^{1}$ it opens up the possibility of computing the soft factor unambiguously by examining purely classical processes, even in four dimensions. More precisely, in four space-time dimensions, the radiative components of the electromagnetic or gravitational field is given in terms of the soft factor $S$ as

$$
-i \frac{1}{4 \pi R} e^{i \omega R} S
$$

Thus for gravitational and electromagnetic fields, knowledge of the classical radiative field at the subleading order defines for us the corresponding soft factor at subleading order. We can then use this to explore the effect of infrared divergences. This is the task we undertake in this paper. We find that while the radiative part of classical fields is well defined in a classical scattering process, the problem appears when we try to carry out a Taylor (more precisely Laurent) series expansion in the frequency $\omega$ of the soft radiation. The leading term of order $\omega^{-1}$ is well defined but at the subleading order there is a term proportional to $\ln \omega$ in four dimensions. This dominates the order unity term that is usually the subleading soft factor in higher dimensions.

One can in fact find a trace of such logarithmic corrections in the standard soft theorem itself. Both for electromagnetism and gravity, the subleading soft theorem has terms proportional to the angular momentum $\mathbf{j}^{\mu \nu}$ of the incoming and outgoing finite energy objects. For a classical particle with trajectory $r^{\mu}(\tau)$, where $\tau$ is the proper time, the orbital part of $\mathbf{j}^{\mu \nu}$ is given by $r^{\mu} p^{\nu}-r^{\nu} p^{\mu}$, where $p^{\mu}=m d r^{\mu} / d \tau$ and $m$ is the mass of the particle. In dimensions higher than four, $r^{\mu}$ grows as $V^{\mu} \tau+c^{\mu}$ for large $|\tau|$, where $V^{\mu}$ and $c^{\mu}$ are constants. It is easy to see that $\mathbf{j}^{\mu \nu}$ computed using this expression is $\tau$ independent and therefore has a finite $\tau \rightarrow \pm \infty$ limit. However in four space-time dimensions, in the large $|\tau|$ limit, $r^{\mu}(\tau)$ will have an additional term proportional to $\ln |\tau|$ due to the long range attractive force due to other particles involved in the scattering. It is easy to verify that $\mathbf{j}^{\mu \nu}$ now acquires terms proportional to $\ln |\tau|$ which do not have finite limit as $\tau \rightarrow \pm \infty$. Therefore the soft theorem itself shows that it breaks down in four space-time dimensions.

A naive guess would be that the logarithmic terms at the subleading order may be given simply by replacing $\ln |\tau|$ by $\ln \omega^{-1}$ in the usual soft theorem. We set out to test this by examining the explicit formula for radiative fields during classical scattering processes. We find that this is indeed true for all cases for which we carry out the analysis.

We now give a summary of our results. The first scattering we analyze is that of a probe of a charge $q$ and mass $m$ from a heavy scatterer of charge $Q$ and mass $M_{0}$ via electromagnetic interaction, and compute the radiative part of the electromagnetic field of polarization $\varepsilon$ and frequency $\omega$ along the direction $\hat{n}$. By comparing this with (1.1) we extract the soft factor in four dimensions. The result takes the form

$$
\tilde{S}_{\mathrm{em}}=-\frac{q}{\omega}\left[\frac{\vec{\varepsilon} \cdot \vec{\beta}_{+}}{1-\hat{n} \cdot \vec{\beta}_{+}}-\frac{\vec{\varepsilon} \cdot \vec{\beta}_{-}}{1-\hat{n} \cdot \vec{\beta}_{-}}\right]-i q \ln \omega^{-1}\left[C_{+} \frac{\vec{\varepsilon} \cdot \vec{\beta}_{+}}{1-\hat{n} \cdot \vec{\beta}_{+}}-C_{-} \frac{\vec{\varepsilon} \cdot \vec{\beta}_{-}}{1-\hat{n} \cdot \vec{\beta}_{-}}\right]+\text {finite }
$$

\footnotetext{
${ }^{1}$ As soft factor beyond the leading order is a function of angular momenta of external states represented as differential operators, by classical soft factor we mean replacing these differential operators by classical angular momenta of external particles.
} 
where $\vec{\beta}_{ \pm}$denotes the velocities $d \vec{r} / d t$ of the probe as $t \rightarrow \pm \infty$, and

$$
C_{ \pm}= \pm \frac{q Q}{4 \pi m\left|\vec{\beta}_{ \pm}\right|^{3}}\left(1-\vec{\beta}_{ \pm}^{2}\right)^{3 / 2}
$$

(1.2) agrees with what we would get by replacing the $\ln |\tau|$ factor in the soft theorem by $\ln \omega^{-1}$.

Next we analyze a similar scattering, but instead of computing emission of electromagnetic wave, we compute the emission of gravitational wave. However we ignore the effect of gravitational force on the scattering, treating gravity at the linearized level sourced by the energy density carried by the probe and the electromagnetic field. By comparing this with (1.1) we extract the following form of the soft graviton factor:

$$
\begin{aligned}
S_{\mathrm{gr}}= & -\frac{m}{\omega} \varepsilon^{i j}\left\{\frac{1}{1-\hat{n} \cdot \vec{\beta}_{+}} \frac{1}{\sqrt{1-\vec{\beta}_{+}^{2}}} \beta_{+i} \beta_{+j}-\frac{1}{1-\hat{n} \cdot \vec{\beta}_{-}} \frac{1}{\sqrt{1-\vec{\beta}_{-}^{2}}} \beta_{-i} \beta_{-j}\right\} \\
& -i m \ln \omega^{-1} \varepsilon^{i j}\left[\frac{1}{\sqrt{1-\vec{\beta}_{+}^{2}}} \beta_{+i} \beta_{+j} C_{+} \frac{1}{1-\hat{n} \cdot \vec{\beta}_{+}}-\frac{1}{\sqrt{1-\vec{\beta}_{-}^{2}}} \beta_{-i} \beta_{-j} C_{-} \frac{1}{1-\hat{n} \cdot \vec{\beta}_{-}}\right] \\
& + \text {finite }
\end{aligned}
$$

with $C_{ \pm}$given by (1.3). This also agrees with what one would get from the soft theorem by replacing the $\ln |\tau|$ factor by $\ln \omega^{-1}$.

Our final example involves scattering of a neutral probe of mass $m$ from a massive scatterer of mass $M_{0}$ via gravitational force in the limit of large impact parameter. For this analysis we take into account the non-linear effects of gravity, e.g. the gravitational field produced by the probe and the scatterer acts as the source of gravity. The soft graviton factor extracted from this analysis takes the same form as (1.4) where now, in the $8 \pi G=1$ unit,

$$
C_{ \pm}=\mp \frac{M_{0}\left(1-3 \vec{\beta}_{ \pm}^{2}\right)}{8 \pi\left|\vec{\beta}_{ \pm}\right|^{3}} .
$$

This again agrees with what we would get by replacing $\ln |\tau|$ by $\ln \omega^{-1}$ in the usual soft theorem.

In the last example there is an additional subtlety that needs some discussion. Since the long range gravitational force acts on the soft graviton as well, the trajectory of the soft graviton far away from the scatterer takes the form $t=R+(4 \pi)^{-1} M_{0} \ln R$. For this reason the radiative component of the gravitational field will be proportional to $\exp [i \omega\{R+$ $\left.\left.(4 \pi)^{-1} M_{0} \ln R-t\right\}\right] / R$ instead of the usual factor $\exp [i \omega(R-t)] / R$. Therefore (1.1) should contain an infrared divergent phase factor of $\exp \left[i \omega \frac{M_{0}}{4 \pi} \ln R\right]$. To this end we would like to remind the reader that the procedure for taking the classical limit, as described in [85], does not fix the overall phase in (1.1); this must be fixed by comparison with explicit results. Comparison with the results of explicit calculation shows that the additional factor is

$$
\exp \left[i \omega \frac{M_{0}}{4 \pi} \ln (\omega R)\right] .
$$


This phase factor, although present, is harmless since this does not affect the flux of soft gravitons, although it can affect the shape of the gravitational wave-form. We expect this to be related to the infrared divergent corrections to the soft factor found in [17], and the classical counterpart of this calculation given in [86-88]. The term proportional to $\ln R$ represents the time delay of a gravitational wave to reach its target at distance $R$ due to the long range gravitational force of the mass $M_{0}$.

Physically the corrections to the soft theorem associated with the $\ln |\tau|$ terms in $\mathbf{j}^{\mu \nu}$ may be understood as the effect of the early and late time acceleration and deceleration of the finite energy particles due to the long range force that they exert on each other. Due to this effect the particles continue to radiate even at large time, producing soft radiation that is responsible for the $\ln \omega^{-1}$ contribution. This interpretation also tells us what the natural scale of the problem is: it is the time-scale beyond which the particle trajectory settles down to the form $x^{\mu}=c^{\mu}+V^{\mu} \tau+a^{\mu} \ln |\tau|$ due to long range electromagnetic or gravitational force. For particle scattering in a Schwarzschild metric this is of the order of the impact parameter $b$, therefore $\ln \omega^{-1}$ stands for $\ln \left(\omega^{-1} b^{-1}\right)$.

Even though the analysis of the paper is restricted to the case of scattering of a light particle by a heavy particle, there is a natural generalization of our result for generic scattering involving multiple initial and final states. Each initial state particle will have early acceleration due to the long range force of the other initial state particles. Similarly each final state particle will have late acceleration due to the long range force of the other final state particles. This will produce terms proportional to $\ln |\tau|$ in the expression for the angular momenta of the particles. The natural generalization of our conjecture will be that in the expression for the subleading soft factor the factors of $\ln |\tau|$ will be replaced by $\ln \omega^{-1}$.

It is natural to ask what these results mean for the quantum theory. As already pointed out, since the S-matrix itself is divergent, in general the soft factor is ambiguous unless the divergences cancel from both sides. The correct approach to studying soft theorem in four space-time dimensions would be to work with finite quantities like inclusive cross section [89-91] or Fadeev-Kulish formalism [92-94], and then see how cross sections / amplitudes with and without soft external states are related. Presumably by taking the classical limit of such modified multiple soft graviton theorem as in [85] we shall reproduce the results of this paper. Furthermore in that analysis the terms proportional to $\ln \omega^{-1}$ would appear directly as $\ln \omega^{-1}$ and there will be no need to make an ad hoc replacement of $\ln |t|$ by $\ln \omega^{-1}$. However derivation of soft theorem in the Faddeev-Kulish formalism is still in its infancy, and even the leading single soft theorem has not been fully understood in this formalism [95]. Therefore derivation of subleading multiple soft theorem, needed for deriving the subleading correction to the classical soft theorem, may not be forthcoming in the near future.

We end this section with a few remarks.

1. The soft factors given in (1.2), (1.4) have finite $\left|\vec{\beta}_{ \pm}\right| \rightarrow 1$ limit if we keep the energies $E_{ \pm}=m / \sqrt{1-\vec{\beta}_{ \pm}^{2}}$ fixed in this limit. Therefore the results are also valid for massless probes. 
2. The leading terms in the soft factors have the property that they vanish in the limit when the deflection goes to zero. This can be seen by setting $\vec{\beta}_{+}=\vec{\beta}_{-}$. This is also the property of the usual subleading factors that arise in higher dimensions. In contrast, the logarithmic terms in (1.2), (1.4) do not vanish in the limit $\vec{\beta}_{+} \rightarrow \vec{\beta}_{-}$ since $C_{ \pm}$have opposite signs. This is a reflection of the fact that the logarithmic terms come from the early and late time acceleration due to the long range force, and this persists even in the absence of any scattering.

3. For real polarizations, the terms proportional to $\ln \omega^{-1}$ in (1.2) and (1.4) are purely imaginary. Therefore they do not contribute to the power spectrum - proportional to $|S|^{2}$ - to subleading order. However for circular polarizations, for which the $\varepsilon$ 's are complex, there may be non-vanishing contribution to the power spectrum at the subleading order, since the tensors that are contracted with the polarizations at the leading and subleading orders are different, and the subleading contribution cannot be factored out as a pure phase.

4. Our analysis also suggests a regime in parameter space where the usual soft expansion may dominate the logarithmic terms. For definiteness let us focus on soft graviton emission. If the scattering takes place via some interaction of range $b$ that is large compared to the Schwarzschild radius $M_{0} /(4 \pi)$ of the scatterer, then for impact parameter of order $b$ and sufficiently large interaction strength - e.g. hard elastic scattering - we can produce appreciable deflection. This would give a leading contribution to $S_{\mathrm{gr}}$ of order $m / \omega$ and the usual subleading soft factor of order $m b$ since the soft expansion parameter is of order $\omega b$. On the other hand the logarithmic term is of order $m M_{0} \ln \omega^{-1}$. Therefore for $b \gg M_{0}$ we can choose a range of $\omega$ in which the soft expansion parameter $\omega b$ is small, but $b \gg M_{0} \ln \omega^{-1}$. In this range the usual soft terms will dominate the logarithmic term. Examples of such scattering can be found in sections 7.2.1 and 7.2.2 of [85].

\section{Logarithmic corrections from soft factors}

In this section we shall see that even the usual soft theorems - valid in dimensions larger than four - develop logarithmic factors when extrapolated to four space-time dimensions. We shall begin by reviewing the results of [85] that relates the soft factor to the radiative component of electromagnetic and gravitational fields. The general relation in $D$-dimensions takes the following form for the gravitational field $\tilde{h}_{\alpha \beta}(\omega, \vec{x})$, related to $h_{\alpha \beta}(t, \vec{x})=\left(g_{\alpha \beta}-\eta_{\alpha \beta}\right) / 2$ by Fourier transform in the time variable:

$$
\begin{aligned}
\tilde{h}_{\alpha \beta}(\omega, \vec{x}) & =\tilde{e}_{\alpha \beta}(\omega, \vec{x})-\frac{1}{D-2} \eta_{\alpha \beta} \tilde{e}_{\gamma}^{\gamma}(\omega, \vec{x}), \\
\varepsilon^{\alpha \beta} \tilde{e}_{\alpha \beta}(\omega, \vec{x}) & =\mathcal{N}^{\prime} S_{\mathrm{gr}}(\varepsilon, k), \\
R \equiv|\vec{x}|, \quad \mathcal{N}^{\prime} & \equiv e^{i \omega R}\left(\frac{\omega}{2 \pi i R}\right)^{(D-2) / 2} \frac{1}{2 \omega}, \quad k \equiv-\omega(1, \hat{n}), \quad \hat{n}=\frac{\vec{x}}{|\vec{x}|} .
\end{aligned}
$$


Here $\varepsilon$ is any arbitrary rank two polarization tensor and $S_{\mathrm{gr}}$ is the soft factor for gravity whose expression will be given in (2.4). A similar formula exists for electromagnetism. The radiative component of the gauge field $\widetilde{A}_{\alpha}(\omega, \vec{x})$, related to the gauge field $A_{\alpha}(t, \vec{x})$ by Fourier transformation in the time variable, is given by

$$
\varepsilon^{\alpha} \widetilde{A}_{\alpha}(\omega, \vec{x})=\mathcal{N}^{\prime} S_{\mathrm{em}}(\varepsilon, k) .
$$

We shall now write down the explicit form of $S_{\mathrm{em}}(\varepsilon, k)$ and $S_{\mathrm{gr}}(\varepsilon, k)$ to subleading order. For simplicity we shall consider the scattering of a pair of particles and work in the probe approximation where one of the objects (the probe) has mass much larger than the other (the scatterer). In this case we have

$$
\begin{aligned}
S_{\mathrm{em}}(\varepsilon, k)= & q \sum_{a=1}^{2}(-1)^{a-1} \frac{\varepsilon \cdot p_{(a)}}{k \cdot p_{(a)}}+i \sum_{a=1}^{2}(-1)^{a-1} q \frac{\varepsilon_{\nu} k_{\rho}}{p_{(a)} \cdot k} \mathbf{j}_{(a)}^{\rho \nu}+\text { non-universal } \\
S_{\mathrm{gr}}(\varepsilon, k)= & \sum_{a=1}^{2}\left[\frac{\varepsilon_{\mu \nu} p_{(a)}^{\mu} p_{(a)}^{\nu}}{p_{(a)} \cdot k}+\varepsilon_{00} \frac{p_{(a)} \cdot k}{\left(k^{0}\right)^{2}}+2 \varepsilon_{0 \nu} \frac{p_{(a)}^{\nu}}{k^{0}}\right] \\
& +i \sum_{a=1}^{2}\left[\left\{\frac{\varepsilon_{\mu \nu} p_{(a)}^{\mu} k_{\rho}}{p_{(a)} \cdot k}+\frac{\varepsilon_{\nu 0} k_{\rho}}{k^{0}}\right\} \mathbf{j}_{(a)}^{\rho \nu}+\frac{\mathbf{J}^{j i}}{M_{0}}\left\{\frac{\varepsilon_{i 0} k_{j} p_{(a)} \cdot k}{\left(k^{0}\right)^{2}}+\frac{\varepsilon_{i \nu} p_{(a)}^{\nu} k_{j}}{k^{0}}\right\}\right] .
\end{aligned}
$$

Here $p_{(1)}$ and $p_{(2)}$ are the momenta of the probe before and after the scattering and $q$ is the charge of the probe. The scatterer is initially taken to be at rest, with mass $M_{0}$ and angular momentum J. The indices $i, j, \cdots$ run over spatial coordinates and the indices $\mu, \nu, \cdots$ run over all space-time coordinates. $\mathbf{j}_{(1)}$ and $\mathbf{j}_{(2)}$ are the angular momenta of the probe before and after the scattering, measured with respect to the space-time point describing the location of the center of momentum of the scatterer at some particular instant of time before the scattering. All momenta and angular momenta are measured with the convention that they are counted with positive sign for ingoing and negative sign for outgoing particles; for charges this is accounted for by the explicit $(-1)^{a}$ factors in $(2.3)$. The indices are raised and lowered by flat metric $\eta^{\mu \nu}$ and $\eta_{\mu \nu}$ with mostly plus signature. The non-universal terms in the soft photon theorem appear at the subleading order but they will not affect our analysis below.

For electromagnetic radiation the radiative part of the field satisfies the constraint equation $k^{\alpha} \widetilde{A}_{\alpha}=0$. This is reflected in the invariance of $S_{\mathrm{em}}(\varepsilon, k)$ under $\varepsilon^{\mu} \rightarrow \varepsilon^{\mu}+k^{\mu}$. Therefore $\widetilde{A}_{i}$ determines $\widetilde{A}_{0}$ and we can focus on the spatial components $\widetilde{A}_{i}$. Consequently we can restrict $\varepsilon^{\alpha}$ to have only spatial components. On the other hand the radiative part of the gravitational field satisfies the constraint $k^{\mu} \tilde{e}_{\mu \nu}=0$, reflected in the invariance of $S_{\mathrm{gr}}(\varepsilon, k)$ under $\varepsilon_{\mu \nu} \rightarrow \varepsilon_{\mu \nu}+\xi_{\mu} k_{\nu}+\xi_{\nu} k_{\mu}$. This allows us to determine $\tilde{e}_{0 \mu}$ in terms of the spatial components $\tilde{e}_{i j}$ and we can focus on the spatial components $\tilde{e}_{i j}$. Consequently we can choose $\varepsilon^{\mu \nu}$ to have only transverse components $\varepsilon^{i j}$. These may be summarized as:

$$
\varepsilon^{0}=0, \quad \varepsilon^{0 \rho}=0
$$

Since both electrodynamics and gravity has gauge symmetries, we can determine the field configurations only up to a choice of gauge. Consequently (2.1) and (2.2) are valid 
only up to gauge transformations:

$$
\begin{aligned}
\delta \tilde{h}_{\mu \nu} & =k_{\mu} \xi_{\nu}+\xi_{\mu} k_{\nu} \Rightarrow \delta \tilde{e}_{\mu \nu}=k_{\mu} \xi_{\nu}+\xi_{\mu} k_{\nu}-\xi . k \eta_{\mu \nu} \\
\delta \widetilde{A}_{\mu} & =\xi k_{\mu}
\end{aligned}
$$

for arbitrary parameters $\xi_{\mu}$ and $\xi$. Using these in (2.1) and (2.2) we see that the physical part of $S_{\mathrm{gr}}(\varepsilon, k)$ and $S_{\mathrm{em}}(\varepsilon, k)$ are contained in those choices of polarization tensor / vector that satisfy

$$
k_{\mu} \varepsilon^{\mu \nu}-\frac{1}{2} k^{\nu} \varepsilon_{\rho}^{\rho}=0, \quad k_{\rho} \varepsilon^{\rho}=0 .
$$

Combining these with (2.5) we get

$$
\varepsilon^{0 \rho}=0, \quad k_{i} \varepsilon^{i j}=0, \quad \varepsilon_{i}^{i}=0, \quad \varepsilon^{0}=0, \quad k_{i} \varepsilon^{i}=0 .
$$

Let $m$ be the mass of the probe particle and $\vec{\beta}_{-}$and $\vec{\beta}_{+}$be its initial and final velocities. Then we have

$$
p_{(1)}=\frac{m}{\sqrt{1-\vec{\beta}_{-}^{2}}}\left(1, \vec{\beta}_{-}\right), \quad p_{(2)}=-\frac{m}{\sqrt{1-\vec{\beta}_{+}^{2}}}\left(1, \vec{\beta}_{+}\right) .
$$

The minus sign in the expression for $p_{(2)}$ is a reflection of the fact that it is an outgoing momentum. Of special interest will be the initial and final trajectories $r_{(1)}(t)$ and $r_{(2)}(t)$. In dimensions $D>4$ these can be taken to be of the form

$$
r_{(1)}^{0}=t, \quad r_{(2)}^{0}=t, \quad \vec{r}_{(1)}=\vec{\beta}_{-} t+\vec{c}_{-}, \quad \vec{r}_{(2)}=\vec{\beta}_{+} t+\vec{c}_{+},
$$

for constant vectors $\vec{c}_{ \pm}$. Therefore we have

$$
\begin{aligned}
& \mathbf{j}_{(1)}^{i j}=r_{(1)}^{i} p_{(1)}^{j}-r_{(1)}^{j} p_{(1)}^{i}=\frac{m}{\sqrt{1-\vec{\beta}_{-}^{2}}}\left(c_{-}^{i} \beta_{-}^{j}-c_{-}^{j} \beta_{-}^{i}\right), \\
& \mathbf{j}_{(1)}^{0 i}=r_{(1)}^{0} p_{(1)}^{i}-r_{(1)}^{i} p_{(1)}^{0}=-\frac{m}{\sqrt{1-\vec{\beta}_{-}^{2}}} c_{-}^{i}, \\
& \mathbf{j}_{(2)}^{i j}=r_{(2)}^{i} p_{(2)}^{j}-r_{(2)}^{j} p_{(2)}^{i}=-\frac{m}{\sqrt{1-\vec{\beta}_{+}^{2}}}\left(c_{+}^{i} \beta_{+}^{j}-c_{+}^{j} \beta_{+}^{i}\right), \\
& \mathbf{j}_{(2)}^{0 i}=r_{(2)}^{0} p_{(2)}^{i}-r_{(2)}^{i} p_{(2)}^{0}=\frac{m}{\sqrt{1-\vec{\beta}_{+}^{2}}} c_{+}^{i} .
\end{aligned}
$$

In particular these approach finite limit as $t \rightarrow \pm \infty$. However in $D=4$ there is a long range force on the incoming and outgoing probe that falls off according to inverse square law. It is easy to verify that in this case the particle trajectories (2.10) are modified to

$$
r_{(1)}^{0}=t, \quad r_{(2)}^{0}=t, \quad \vec{r}_{(1)}=\vec{\beta}_{-} t+\vec{c}_{-}-C_{-} \vec{\beta}_{-} \ln |t|, \quad \vec{r}_{(2)}=\vec{\beta}_{+} t+\vec{c}_{+}-C_{+} \vec{\beta}_{+} \ln |t|,
$$

for appropriate constants $C_{ \pm}$. This modifies the expressions for $\mathbf{j}_{(i)}^{\mu \nu}$ to

$$
\begin{array}{ll}
\mathbf{j}_{(1)}^{i j}=\frac{m}{\sqrt{1-\vec{\beta}_{-}^{2}}}\left(c_{-}^{i} \beta_{-}^{j}-c_{-}^{j} \beta_{-}^{i}\right), & \mathbf{j}_{(1)}^{0 i}=-\frac{m}{\sqrt{1-\vec{\beta}_{-}^{2}}}\left\{c_{-}^{i}-C_{-} \beta_{-}^{i} \ln |t|\right\}, \\
\mathbf{j}_{(2)}^{i j}=-\frac{m}{\sqrt{1-\vec{\beta}_{+}^{2}}}\left(c_{+}^{i} \beta_{+}^{j}-c_{+}^{j} \beta_{+}^{i}\right), & \mathbf{j}_{(2)}^{0 i}=\frac{m}{\sqrt{1-\vec{\beta}_{+}^{2}}}\left\{c_{+}^{i}-C_{+} \beta_{+}^{i} \ln |t|\right\} .
\end{array}
$$


Note in particular that $\mathbf{j}_{(a)}^{0 j}$ diverges as $|t| \rightarrow \infty$, making the expressions ill-defined. Ignoring this for the time being, for the particle kinematics described above we can express the soft factors given in (2.3) and (2.4) as

$$
S_{\mathrm{em}}=-\frac{q}{\omega}\left[\frac{\vec{\varepsilon} \cdot \vec{\beta}_{+}}{1-\hat{n} \cdot \vec{\beta}_{+}}-\frac{\vec{\varepsilon} \cdot \vec{\beta}_{-}}{1-\hat{n} \cdot \vec{\beta}_{-}}\right]-i q \ln |t|\left[C_{+} \frac{\vec{\varepsilon} \cdot \vec{\beta}_{+}}{1-\hat{n} \cdot \vec{\beta}_{+}}-C_{-} \frac{\vec{\varepsilon} \cdot \vec{\beta}_{-}}{1-\hat{n} \cdot \vec{\beta}_{-}}\right]+\text {finite }
$$

and

$$
\begin{aligned}
S_{\mathrm{gr}}= & -\frac{m}{\omega} \varepsilon^{i j}\left\{\frac{1}{1-\hat{n} \cdot \vec{\beta}_{+}} \frac{1}{\sqrt{1-\vec{\beta}_{+}^{2}}} \beta_{+i} \beta_{+j}-\frac{1}{1-\hat{n} \cdot \vec{\beta}_{-}} \frac{1}{\sqrt{1-\vec{\beta}_{-}^{2}}} \beta_{-i} \beta_{-j}\right\} \\
& -i m \ln |t| \varepsilon^{i j}\left[\frac{1}{\sqrt{1-\vec{\beta}_{+}^{2}}} \beta_{+i} \beta_{+j} C_{+} \frac{1}{1-\hat{n} \cdot \vec{\beta}_{+}}-\frac{1}{\sqrt{1-\vec{\beta}_{-}^{2}}} \beta_{-i} \beta_{-j} C_{-} \frac{1}{1-\hat{n} \cdot \vec{\beta}_{-}}\right] \\
& + \text {finite }
\end{aligned}
$$

where 'finite' refers to terms which remain finite as $\omega \rightarrow 0,|t| \rightarrow \infty$.

A natural guess is that the presence of $\ln |t|$ term implies the breakdown of the expansion of the soft factor in power series in $\omega$. Naively one might expect that the correct expression is given by replacing the $\ln |t|$ factors by $\ln \omega^{-1}$. In the following we shall verify this by explicit computation in several examples.

\section{Some relevant integrals}

In our analysis we shall often encounter integrals of the form

$$
I=\int d t e^{i \omega g(t)} F(t)+\text { boundary terms }
$$

where $g(t)$ and $F(t)$ are functions of $t$ and the integration over $t$ runs from $-\infty$ to $+\infty$. As will be discussed shortly, the 'boundary terms' need to be adjusted to make the integral well-defined. In all the examples considered, $g(t)$ will grow as $a_{ \pm} t$ as $t \rightarrow \pm \infty$ for some constants $a_{ \pm}$, with possible corrections of order $\ln |t| . F(t)$ will typically either approach a constant or fall off as some negative power of $t$, again with possible subleading corrections involving $\ln |t|$. If $F(t) \sim|t|^{-\alpha}$ for $\alpha>0$, then the integral is well defined, and can be evaluated by taking the limits to be from $-T$ to $T$ and taking the $T \rightarrow \infty$ limit. If on the other hand $F(t) \sim|t|^{-\alpha}$ with $-1<\alpha \leq 0$, then we have to define the integral by first performing an integration by parts:

$$
I=\int d t\left\{\frac{d}{d t} e^{i \omega g(t)}\right\} \frac{1}{i \omega g^{\prime}(t)} F(t)+\text { boundary terms }=-\frac{1}{i \omega} \int d t e^{i \omega g(t)} \frac{d}{d t}\left\{\frac{F(t)}{g^{\prime}(t)}\right\},
$$

where the boundary terms have been chosen to cancel the boundary terms arising from integration by parts. Since $g^{\prime}(t) \rightarrow a_{ \pm}$as $t \rightarrow \pm \infty$ and $F(t) \sim|t|^{-\alpha}$ as $t \rightarrow \pm \infty$, the integrand in (3.2) falls off as $t^{-\alpha-1}$ and therefore for $\alpha>-1$ this can be defined by putting 
limits $\pm T$ on the $t$ integral and then taking the limit $T \rightarrow \infty$. This makes the integral welldefined without boundary terms. If $\alpha \leq-1$ then we need to carry out more integration by parts but we shall not encounter such a situation.

Once we have defined the integral so that it can be evaluated as limits of an integral with finite range, we are free to go back to the original form by integration by parts, but now we have to keep track of the boundary terms. This reduces the integral to

$$
I=\lim _{T \rightarrow \infty}\left\{\int_{-T}^{T} d t e^{i \omega g(t)} F(t)-\frac{1}{i \omega}\left[e^{i \omega g(t)} \frac{F(t)}{g^{\prime}(t)}\right]_{-T}^{T}\right\} .
$$

We can use either the right hand side of (3.2) or (3.3) as the proper definition of (3.1) after taking the $T \rightarrow \infty$ limit. The first term in (3.3) has less powers of $\omega$ in the denominator compared to the right hand side of (3.2), but the boundary terms carry powers of $\omega$ in the denominator and provide the missing terms. Therefore (3.3) is convenient for carrying out a small $\omega$ expansion. This can be done by first carrying out the small $\omega$ expansion of (3.3) and then taking the limit $T \rightarrow \infty$. This is the strategy that was used in [85] for checking soft theorems in dimensions $D>4$.

This procedure is useful if the expansion does not have terms of order $\ln \omega^{-1}$, but runs into difficulty if there are $\ln \omega^{-1}$ terms in the expansion. To see how this happens, note that the right hand side of (3.2) is well defined as integral over a finite range $(-T, T)$ and also in the limit $T \rightarrow \infty$. Therefore (3.3) is also well-defined for $T \rightarrow \pm \infty$. However suppose that we take the form given in (3.3) and carry out the expansion in $\omega$ before taking the $T \rightarrow \infty$ limit, and then in each term in the expansion take the $T \rightarrow \infty$ limit. Since this will always produce power series in $\omega$, the only way we can see the presence of the $\ln \omega^{-1}$ term is that the expansion coefficients will now fail to have finite limit as $T \rightarrow \infty$ even though the original expressions (3.2) and (3.3) have well defined $T \rightarrow \infty$ limit.

We shall analyze the logarithmic terms in the soft factor by always working with the convergent form of the integral as in the right hand side of (3.2) without explicit boundary terms and then analyzing the behavior of the integral in the $\omega \rightarrow 0$ limit without first naively expanding the integrand in powers of $\omega$. Below we write down the expressions of five different types of integrals that we shall need for our analysis and the values of the integrals for small $\omega$. The derivation can be found in appendix A.

Let $f, g, h$ and $r$ be functions of $t$ with the following asymptotic behavior:

$$
\begin{array}{ll}
f(t) \rightarrow f_{ \pm}+\frac{k_{ \pm}}{t}, & g(t) \rightarrow a_{ \pm} t+b_{ \pm} \ln |t|, \\
h(t) \rightarrow p_{ \pm} t+q_{ \pm} \ln |t|, & r(t) \rightarrow c_{ \pm} t+d_{ \pm} \ln |t|, \quad \text { as } \quad t \rightarrow \pm \infty .
\end{array}
$$

In appendix $\mathrm{A}$ we prove the following results for arbitrary constant $R$ :

$$
\begin{aligned}
& I_{1} \equiv \frac{1}{\omega} \int_{-\infty}^{\infty} d t e^{-i \omega g(t)} f^{\prime}(t)=\omega^{-1}\left(f_{+}-f_{-}\right)+i\left(a_{+} k_{+}-a_{-} k_{-}\right) \ln \omega^{-1}+\text { finite }, \\
& I_{2} \equiv \int_{-\infty}^{\infty} d t e^{-i \omega g(t)} \frac{d}{d t}\left[f(t)\left\{\ln \frac{h(t)}{R}+\int_{h(t)}^{\infty} e^{i \omega u} \frac{d u}{u}\right\}\right]=-\left(f_{+}-f_{-}\right) \ln (R \omega)+\text { finite },
\end{aligned}
$$




$$
\begin{aligned}
I_{3} & \equiv \int_{-\infty}^{\infty} d t \frac{1}{r(t)} f(t)\left[e^{-i \omega g(t)}-e^{-i \omega h(t)}\right]=\text { finite } \\
I_{4} & \equiv \frac{1}{\omega} \int_{-\infty}^{\infty} d t \frac{1}{r(t)^{2}} f(t)\left[e^{-i \omega g(t)}-e^{-i \omega h(t)}\right] \\
& =-i\left\{f_{+} c_{+}^{-2}\left(a_{+}-p_{+}\right)-f_{-} c_{-}^{-2}\left(a_{-}-p_{-}\right)\right\} \ln \omega^{-1}+\text { finite } \\
I_{5} & \equiv \int_{-\infty}^{\infty} d t \frac{1}{r(t)} f(t) e^{-i \omega g(t)}=\left(f_{+} c_{+}^{-1}-f_{-} c_{-}^{-1}\right) \ln \omega^{-1}+\text { finite }
\end{aligned}
$$

\section{Electromagnetic radiation}

In this section we shall analyze the electromagnetic radiation due to the scattering of a charged probe from a charged scatterer. This is given by [85]

$$
\widetilde{A}_{\alpha}(\omega, \vec{x})=i \mathcal{N}^{\prime} \int d \sigma e^{i \omega\left\{r^{0}(\sigma)-\hat{n} \cdot \vec{r}(\sigma)\right\}} q V_{\alpha}(\sigma)+\text { boundary terms },
$$

where the 'boundary terms' are determined using the principle described in section 3, $\hat{n}=\vec{x} /|\vec{x}|, \sigma$ denotes the proper time along the particle trajectory $r(\sigma)$ and $V^{\alpha}$ is the four velocity

$$
V^{\alpha}(\sigma)=\frac{d r^{\alpha}}{d \sigma}
$$

It can be shown that [85] in four space-time dimensions (4.1) reduces to the standard formula for electromagnetic radiation from an accelerated particle described e.g. in [96]. By a change of variables from $\sigma$ to $t=r^{0}$ and an integration by parts, we can bring this expression into the form

$$
\widetilde{A}_{i}(\omega, \vec{x})=-q \omega^{-1} \mathcal{N}^{\prime} \int d t e^{i \omega\{t-\hat{n} \cdot \vec{r}(t)\}} \frac{d}{d t}\left\{\frac{1}{1-\hat{n} \cdot \vec{v}(t)} v_{i}(t)\right\}
$$

where we have focussed on the spatial components of $\widetilde{A}$. Since it follows from (2.12) that $\vec{v}(t)=d \vec{r} / d t$ approaches a constant plus terms of order $1 /|t|$ for large $t$, the integrand in (4.3) falls off as $1 / t^{2}$ and therefore we do not need to add boundary terms in this representation.

Comparing (4.3) with (2.2) we can identify the prediction for the soft factor from classical analysis:

$$
\tilde{S}_{\mathrm{em}}(\varepsilon, k)=-\frac{q}{\omega} \int_{-\infty}^{\infty} d t e^{i \omega(t-\hat{n} \cdot \vec{r}(t))} \frac{d}{d t}\left[\frac{\vec{\varepsilon} \cdot \vec{v}}{1-\hat{n} . \vec{v}}\right]
$$

assuming that $\varepsilon$ has only spatial components. Now as $t \rightarrow \pm \infty$, we have

$$
\vec{r}(t) \rightarrow \vec{\beta}_{ \pm} t-C_{ \pm} \vec{\beta}_{ \pm} \ln |t|+\text { finite, } \quad \vec{v}(t) \equiv \frac{d \vec{r}(t)}{d t} \rightarrow \vec{\beta}_{ \pm}\left(1-C_{ \pm} t^{-1}\right) .
$$

Therefore the integral in (4.4) has the form of $I_{1}$ given in (3.5) with

$$
\begin{aligned}
& g(t) \equiv \hat{n} \cdot \vec{r}(t)-t \simeq\left(\hat{n} \cdot \vec{\beta}_{ \pm}-1\right) t-C_{ \pm} \hat{n} \cdot \vec{\beta}_{ \pm} \ln |t|+\text { finite } \\
& f(t) \equiv-q\left[\frac{\vec{\varepsilon} \cdot \vec{v}(t)}{1-\hat{n} \cdot \vec{v}(t)}\right]=-q \frac{\vec{\varepsilon} \cdot \vec{\beta}_{ \pm}}{1-\hat{n} \cdot \vec{\beta}_{ \pm}}\left[1-\frac{C_{ \pm}}{1-\hat{n} \cdot \vec{\beta}_{ \pm}} t^{-1}+\cdots\right], \quad \text { as } t \rightarrow \pm \infty .
\end{aligned}
$$


Comparing (4.6) with (3.4) we get

$$
a_{ \pm}=\left(\hat{n} \cdot \beta_{ \pm}-1\right), \quad f_{ \pm}=-q \frac{\vec{\varepsilon} \cdot \vec{\beta}_{ \pm}}{1-\hat{n} \cdot \vec{\beta}_{ \pm}}, \quad k_{ \pm}=q C_{ \pm} \frac{\vec{\varepsilon} \cdot \vec{\beta}_{ \pm}}{\left(1-\hat{n} \cdot \vec{\beta}_{ \pm}\right)^{2}} .
$$

Therefore we get from (3.5)

$$
\tilde{S}_{\mathrm{em}}=-\frac{q}{\omega}\left[\frac{\vec{\varepsilon} \cdot \vec{\beta}_{+}}{1-\hat{n} \cdot \vec{\beta}_{+}}-\frac{\vec{\varepsilon} \cdot \vec{\beta}_{-}}{1-\hat{n} \cdot \vec{\beta}_{-}}\right]-i q \ln \omega^{-1}\left[C_{+} \frac{\vec{\varepsilon} \cdot \vec{\beta}_{+}}{1-\hat{n} \cdot \vec{\beta}_{+}}-C_{-} \frac{\vec{\varepsilon} \cdot \vec{\beta}_{-}}{1-\hat{n} \cdot \vec{\beta}_{-}}\right]+\text {finite }
$$

This agrees with $S_{\text {em }}$ given in (2.14) if we replace $\ln |t|$ by $\ln \omega^{-1}$.

For completeness let us compute $C_{ \pm}$. Asymptotically we can regard the velocity carried by the probe to be in the radial direction. If $Q$ denotes the charge carried by the scatterer, then energy conservation gives

$$
\frac{m}{\sqrt{1-\vec{v}(t)^{2}}}+\frac{q Q}{4 \pi|\vec{r}(t)|}=\text { constant }
$$

Substituting (4.5) into this equation and setting the coefficient of the $1 /|t|$ term for large $|t|$ to zero, we get

$$
C_{ \pm}= \pm \frac{q Q}{4 \pi m\left|\vec{\beta}_{ \pm}\right|^{3}}\left(1-\vec{\beta}_{ \pm}^{2}\right)^{3 / 2}
$$

\section{Gravitational radiation}

In this section we shall analyze the logarithmic correction to the soft factor for gravitational radiation. We shall analyze two examples. In the first the scattering takes place via electromagnetic interaction and the energy momentum tensor during the scattering is used as a source for gravitational radiation. Assuming that the electromagnetic interaction is much stronger than the gravitational interaction during the scattering, we ignore the effect of gravity on the motion of the probe. Therefore for this problem, the non-linear effects of gravity are suppressed. The second example involves the scattering of a neutral probe off a massive object via gravitational interaction. For this problem the non-linear effects of gravity become important since the gravitational field itself acts as a source of gravitational radiation.

\subsection{Gravitational radiation from scattering via electromagnetic interaction}

The set up here is as follows. The probe has mass $m$ and charge $q$ and the scatterer has mass $M_{0} \gg m$ and charge $Q \gg q$. We assume that the distance of closest approach between the probe and the scatterer is large compared to the Schwarzschild radius of the scatterer so that the effect of gravity on the scattering can be ignored, but that $Q$ and $q$ are sufficiently large so that there is appreciable scattering due to the electromagnetic force. In this case the energy momentum tensor, that acts as the source of gravitational radiation, receives contribution from two sources - the probe and the electromagnetic field. As long as we focus on the spacial components of $\tilde{e}_{i j}$ whose source is the spatial component of the 
energy momentum tensor, we can ignore the contribution due to the scatterer due to the smallness of its velocity during the scattering. Consequently the result for $\tilde{e}_{i j}$ is given by the sum of two terms: $\tilde{e}_{i j}^{(1)}$ due to the probe and $\tilde{e}_{i j}^{(2)}$ due to the electromagnetic field. We shall now analyze each component separately.

The radiative part of the gravitational field due to the probe is given by [85]

$$
\tilde{e}_{i j}^{(1)}=i \mathcal{N}^{\prime} \int d \sigma e^{i k . r(\sigma)} P_{i}(\sigma) V_{j}(\sigma)+\text { boundary terms },
$$

where the integral runs over the proper time $\sigma$ along the world-line $r(\sigma)$ of the probe, $V^{\alpha}=d r^{\alpha} / d \sigma$ is the $D$-velocity of the probe and $P^{\alpha}=m V^{\alpha}$ is the momentum of the probe. We now change the integration variable from $\sigma$ to $t=r^{0}$ to express (5.1) as

$$
\tilde{e}_{i j}^{(1)}=i \mathcal{N}^{\prime} \int d t e^{i k . r}\left(\frac{d t}{d \sigma}\right)^{-1} P_{i} V_{j}+\text { boundary terms . }
$$

Using

$$
\begin{aligned}
\left(k^{0}, \vec{k}\right) & =-\omega(1, \vec{n}), \\
V^{0} & =\frac{d r^{0}}{d \sigma}=\frac{d t}{d \sigma}=\frac{1}{\sqrt{1-\vec{v}^{2}}}, \quad \vec{v}(t) \equiv \frac{d \vec{r}}{d t}, \\
\vec{V} & =\frac{d \vec{r}}{d \sigma}=\frac{d t}{d \sigma} \frac{d \vec{r}}{d t}=\frac{1}{\sqrt{1-\vec{v}^{2}}} \vec{v}(t),
\end{aligned}
$$

and

$$
\vec{P}=m \vec{V}=\frac{m}{\sqrt{1-\vec{v}^{2}}} \vec{v}
$$

we can express (5.2) as

$$
\begin{aligned}
\tilde{e}_{i j}^{(1)} & =i \mathcal{N}^{\prime} m \int d t e^{i \omega(t-\vec{n} \cdot \vec{r}(t))} \frac{1}{\sqrt{1-v^{2}}} v_{i} v_{j}+\text { boundary terms } \\
& =i \mathcal{N}^{\prime} m \int d t \frac{1}{i \omega(1-\vec{n} \cdot \vec{v})} \frac{d}{d t}\left(e^{i \omega(t-\vec{n} \cdot \vec{r}(t))}\right) \frac{1}{\sqrt{1-v^{2}}} v_{i} v_{j}+\text { boundary terms } \\
& =-\mathcal{N}^{\prime} m \omega^{-1} \int d t e^{i \omega(t-\vec{n} \cdot \vec{r}(t))} \frac{d}{d t}\left\{\frac{1}{(1-\vec{n} \cdot \vec{v})} \frac{1}{\sqrt{1-v^{2}}} v_{i} v_{j}\right\}
\end{aligned}
$$

Note that since $\vec{v}$ approaches a constant plus terms of order $1 /|t|$ for large $t$, the integral in the last line is convergent and we do not need to add any boundary terms. In this case $\vec{r}(t)$ has the form given in (4.5) as $t \rightarrow \pm \infty$, with $C_{ \pm}$given by (4.10), since in our approximation the long range force between the probe and the scatterer is purely electromagnetic. Therefore in these limits,

$$
\begin{aligned}
& \frac{1}{1-\hat{n} \cdot \vec{v}(t)} \frac{1}{\sqrt{1-\vec{v}^{2}}} v_{i} v_{j} \\
& \simeq \frac{1}{1-\hat{n} \cdot \overrightarrow{\beta_{ \pm}}} \frac{1}{\sqrt{1-\vec{\beta}_{ \pm}^{2}}} \beta_{ \pm i} \beta_{ \pm j}\left[1-\frac{1}{t}\left\{C_{ \pm} \frac{\hat{n} \cdot \vec{\beta}_{ \pm}}{1-\hat{n} \cdot \vec{\beta}_{ \pm}}+C_{ \pm} \frac{\vec{\beta}_{ \pm}^{2}}{1-\vec{\beta}_{ \pm}^{2}}+2 C_{ \pm}\right\}\right], \\
&t-\hat{n} \cdot \vec{r}(t)) \simeq t\left(1-\hat{n} \cdot \vec{\beta}_{ \pm}\right)+C_{ \pm} \hat{n} \cdot \vec{\beta}_{ \pm} \ln |t| .
\end{aligned}
$$


Therefore we can use the formula for $I_{1}$ to express $\tilde{e}_{i j}^{(1)}$ given in (5.7) as

$$
\begin{aligned}
& \tilde{e}_{i j}^{(1)}=-\omega^{-1} m \mathcal{N}^{\prime}\left\{\frac{1}{1-\hat{n} \cdot \vec{\beta}_{+}} \frac{1}{\sqrt{1-\vec{\beta}_{+}^{2}}} \beta_{+i} \beta_{+j}-\frac{1}{1-\hat{n} \cdot \vec{\beta}_{-}} \frac{1}{\sqrt{1-\vec{\beta}_{-}^{2}}} \beta_{-i} \beta_{-j}\right\} \\
& -i m \mathcal{N}^{\prime} \ln \omega^{-1}\left[\frac{1}{\sqrt{1-\vec{\beta}_{+}^{2}}} \beta_{+i} \beta_{+j}\left\{C_{+} \frac{1}{1-\hat{n} \cdot \vec{\beta}_{+}}+C_{+} \frac{1}{1-\vec{\beta}_{+}^{2}}\right\}\right. \\
& \left.-\frac{1}{\sqrt{1-\vec{\beta}_{-}^{2}}} \beta_{-i} \beta_{-j}\left\{C_{-} \frac{1}{1-\hat{n} \cdot \vec{\beta}_{-}}+C_{-} \frac{1}{1-\vec{\beta}_{-}^{2}}\right\}\right] \text {. }
\end{aligned}
$$

We now turn to the contribution $\tilde{e}^{(2)}$ produced by the electromagnetic field. The dominant part of the stress tensor comes from the term proportional to $Q^{2}$, but since the electric field produced by the scatterer is stationary, it does not generate any radiative component. Therefore we focus on the next term proportional to $q Q$. Denoting by $F_{\mu \nu}^{P}(x)$ and $F_{\mu \nu}^{S}(x)$ the field strengths produced by the probe and scatterer respectively, and by $\widetilde{F}_{\mu \nu}^{P}(\omega, \vec{\ell})$ and $\widetilde{F}_{\mu \nu}^{S}(\omega, \vec{\ell})$ their Fourier transform in the space and time variables, we have [85]

$$
\widetilde{F}_{i 0}^{S}(\ell)=-i \ell_{i} Q \frac{1}{\overrightarrow{\ell^{2}}} 2 \pi \delta\left(\ell^{0}\right),
$$

and

$$
\widetilde{F}_{i 0}^{P}(-\ell)=-q \frac{1}{\left(\ell^{0}-i \epsilon\right)^{2}-\overrightarrow{\ell^{2}}} \int d \sigma e^{i \ell \cdot r(\sigma)}\left\{-i \ell_{i} \frac{d r_{0}}{d \sigma}+i \ell_{0} \frac{d r_{i}}{d \sigma}\right\}
$$

Then in $D$ dimensions $\tilde{e}_{i j}^{(2)}$ is given by [85]

$$
\begin{aligned}
\tilde{e}_{i j}^{(2)} & =i \mathcal{N}^{\prime} \int d^{D} x^{\prime} e^{i k \cdot x^{\prime}}\left[-F_{i 0}^{P}\left(x^{\prime}\right) F_{j 0}^{S}\left(x^{\prime}\right)-F_{j 0}^{P}\left(x^{\prime}\right) F_{i 0}^{S}\left(x^{\prime}\right)+\delta_{i j} F_{k 0}^{S}\left(x^{\prime}\right) F_{k 0}^{P}\left(x^{\prime}\right)\right] \\
& =i \mathcal{N}^{\prime} \int \frac{d^{D} \ell}{(2 \pi)^{D}}\left[-\widetilde{F}_{i 0}^{P}(-\ell-k) \widetilde{F}_{j 0}^{S}(\ell)-\widetilde{F}_{j 0}^{P}(-\ell-k) \widetilde{F}_{i 0}^{S}(\ell)+\delta_{i j} \widetilde{F}_{k 0}^{P}(-\ell-k) \widetilde{F}_{k 0}^{S}(\ell)\right]
\end{aligned}
$$

Using (5.10), (5.11) this may be rewritten as

$$
\begin{aligned}
\tilde{e}_{i j}^{(2)}= & i \mathcal{N}^{\prime} \int d \sigma \int \frac{d^{D-1} \ell}{(2 \pi)^{D-1}} e^{i \vec{\ell} \cdot \vec{r}(\sigma)+i k \cdot r(\sigma)} q Q \frac{1}{\left(\overrightarrow{\ell^{2}}\right)\left(\overrightarrow{\ell^{2}}+2 \vec{\ell} \cdot \vec{k}\right)} \\
& {\left[\left\{2 \ell_{i} \ell_{j}+\ell_{i} k_{j}+\ell_{j} k_{i}-\left(\overrightarrow{\ell^{2}}+\vec{\ell} \cdot \vec{k}\right) \delta_{i j}\right\} \frac{d r_{0}}{d \sigma}+\left\{-k_{0} \ell_{j} \frac{d r_{i}}{d \sigma}-k_{0} \ell_{i} \frac{d r_{j}}{d \sigma}+k_{0} \ell_{m} \frac{d r_{m}}{d \sigma} \delta_{i j}\right\}\right] } \\
= & i \mathcal{N}^{\prime} \int d \sigma \int \frac{d^{D-1} \ell}{(2 \pi)^{D-1}} e^{i \vec{\ell} \cdot \vec{r}(\sigma)+i k \cdot r(\sigma)} q Q \frac{1}{\left(\overrightarrow{\ell^{2}}\right)^{2}}\left\{2 \ell_{i} \ell_{j}-\vec{\ell}^{2} \delta_{i j}\right\} \frac{d r_{0}}{d \sigma}+\tilde{f}_{i j}
\end{aligned}
$$




$$
\begin{aligned}
\tilde{f}_{i j} \equiv i \mathcal{N}^{\prime} \int d \sigma \int \frac{d^{D-1} \ell}{(2 \pi)^{D-1}} e^{i \vec{\ell} \cdot \vec{r}(\sigma)+i k \cdot r(\sigma)} q Q \frac{1}{\left(\overrightarrow{\ell^{2}}\right)^{2}\left(\overrightarrow{\ell^{2}}+2 \vec{\ell} \cdot \vec{k}\right)} \\
\\
\left\{-4 \ell_{i} \ell_{j} \vec{\ell} \cdot \vec{k}+\vec{\ell}^{2}\left(\ell_{i} k_{j}+\ell_{j} k_{i}\right)+\vec{\ell} \cdot \vec{k} \vec{\ell}^{2} \delta_{i j}\right\} \frac{d r_{0}}{d \sigma} \\
+i \mathcal{N}^{\prime} \int d \sigma \int \frac{d^{D-1} \ell}{(2 \pi)^{D-1}} e^{i \vec{\ell} \cdot \vec{r}(\sigma)+i k \cdot r(\sigma)} q Q \frac{1}{\left(\overrightarrow{\ell^{2}}\right)\left(\overrightarrow{\ell^{2}}+2 \vec{\ell} \cdot \vec{k}\right)} \\
\left\{-k_{0} \ell_{j} \frac{d r_{i}}{d \sigma}-k_{0} \ell_{i} \frac{d r_{j}}{d \sigma}+k_{0} \ell_{m} \frac{d r_{m}}{d \sigma} \delta_{i j}\right\} .
\end{aligned}
$$

In the expression for $\tilde{f}_{i j}$ the integration over $\ell$ is free from infrared divergence for $D \geq 4$ even after we factor out a power of $\omega$ and then take the $k \rightarrow 0$ limit. Furthermore $d r_{0} / d \sigma$ and $d r_{i} / d \sigma$ approach finite values as $\sigma \rightarrow \pm \infty$. Taking into account the explicit factor of $k$ in all the terms in $\tilde{f}_{i j}$, we have

$$
\tilde{f}_{i j}=\omega \int d t e^{i k \cdot r(t)} f(t)+\text { boundary terms }
$$

where $f(t)$ approaches a finite value as $t \rightarrow \pm \infty$. Rewriting this as

$$
-\int d t e^{i k \cdot r(t)} \frac{d}{d t}\left\{\frac{1}{i(1-\hat{n} \cdot \vec{v})} f(t)\right\}
$$

we see that this has the form $\omega I_{1}$. Therefore it does not have any divergent contribution in the $\omega \rightarrow 0$ limit and we can focus on the contribution to $\tilde{e}_{i j}^{(2)}$ from the first term on the right hand side of (5.13).

Using

$$
\frac{1}{\left(\overrightarrow{\ell^{2}}\right)^{2}}\left\{2 \ell_{i} \ell_{j}-\vec{\ell}^{2} \delta_{i j}\right\}=-\frac{1}{2}\left[\frac{\partial}{\partial \ell_{i}}\left(\frac{\ell_{j}}{\overrightarrow{\ell^{2}}}\right)+\frac{\partial}{\partial \ell_{j}}\left(\frac{\ell_{i}}{\overrightarrow{\ell^{2}}}\right)\right]
$$

and integration by parts, we can express (5.13) as

$$
\begin{aligned}
\tilde{e}_{i j}^{2} & \simeq \frac{i}{2} \mathcal{N}^{\prime} \int d \sigma \int \frac{d^{D-1} \ell}{(2 \pi)^{D-1}} e^{i \vec{\ell} \cdot \vec{r}(\sigma)+i k . r(\sigma)} q Q \frac{1}{\overrightarrow{\ell^{2}}}\left\{i \ell_{i} r_{j}+i \ell_{j} r_{i}\right\} \frac{d r_{0}}{d \sigma} \\
& =-\frac{i}{2} \mathcal{N}^{\prime} \int d \sigma \int \frac{d^{D} \ell}{(2 \pi)^{D}} e^{i \ell . r(\sigma)+i k . r(\sigma)}\left\{q \widetilde{F}_{i 0}^{S}(\ell) r_{j}+q \widetilde{F}_{j 0}^{S}(\ell) r_{i}\right\} \frac{d r_{0}}{d \sigma} \\
& =-\frac{i}{2} \mathcal{N}^{\prime} \int d \sigma e^{i k . r(\sigma)}\left\{q F_{i 0}^{S}(r(\sigma)) r_{j}(\sigma)+q F_{j 0}^{S}(r(\sigma)) r_{i}(\sigma)\right\} \frac{d r_{0}}{d \sigma},
\end{aligned}
$$

where in the second step we have used (5.10). Using equations of motion

$$
\frac{d P_{\alpha}}{d \sigma}=q F_{\alpha \rho}^{S}(r(\sigma)) \frac{d r^{\rho}}{d \sigma},
$$

and the identification $r_{0}=-r^{0}=-t$, we can express (5.18) as

$$
\begin{aligned}
\tilde{e}_{i j}^{(2)} & =\frac{i}{2} \mathcal{N}^{\prime} \int d \sigma e^{i k . r(\sigma)}\left\{\frac{d P_{i}}{d \sigma} r_{j}(\sigma)+\frac{d P_{j}}{d \sigma} r_{i}(\sigma)\right\} \\
& =i \frac{m}{2} \mathcal{N}^{\prime} \int d t e^{i k \cdot r}\left[\frac{d}{d t}\left\{\frac{v_{i}}{\sqrt{1-\vec{v}^{2}}}\right\} r_{j}+\frac{d}{d t}\left\{\frac{v_{j}}{\sqrt{1-\vec{v}^{2}}}\right\} r_{i}\right] .
\end{aligned}
$$


Now specializing to the case $D=4$ and using (4.5) we see that the term inside the square bracket behaves in the limit $t \rightarrow \pm \infty$, as

$$
2 t^{-1} C_{ \pm} \beta_{ \pm i} \beta_{ \pm j} \frac{1}{\left(1-\vec{\beta}_{ \pm}^{2}\right)^{3 / 2}}+\mathcal{O}\left(t^{-2} \ln |t|\right) .
$$

Therefore the integral has the structure of $I_{5}$ and can be evaluated as

$$
\tilde{e}_{i j}^{(2)}=i m \mathcal{N}^{\prime} \ln \omega^{-1}\left[C_{+} \beta_{+i} \beta_{+j} \frac{1}{\left(1-\vec{\beta}_{+}^{2}\right)^{3 / 2}}-C_{-} \beta_{-i} \beta_{-j} \frac{1}{\left(1-\vec{\beta}_{-}^{2}\right)^{3 / 2}}\right] .
$$

Adding (5.9) and (5.22) we get

$$
\begin{aligned}
\tilde{e}_{i j}= & \tilde{e}_{i j}^{(1)}+\tilde{e}_{i j}^{(2)} \\
= & -\omega^{-1} m \mathcal{N}^{\prime}\left\{\frac{1}{1-\hat{n} \cdot \vec{\beta}_{+}} \frac{1}{\sqrt{1-\vec{\beta}_{+}^{2}}} \beta_{+i} \beta_{+j}-\frac{1}{1-\hat{n} \cdot \vec{\beta}_{-}} \frac{1}{\sqrt{1-\vec{\beta}_{-}^{2}}} \beta_{-i} \beta_{-j}\right\} \\
& -i m \mathcal{N}^{\prime} \ln \omega^{-1}\left[\frac{1}{\sqrt{1-\vec{\beta}_{+}^{2}}} \beta_{+i} \beta_{+j} C_{+} \frac{1}{1-\hat{n} \cdot \vec{\beta}_{+}}-\frac{1}{\sqrt{1-\vec{\beta}_{-}^{2}}} \beta_{-i} \beta_{-j} C_{-} \frac{1}{1-\hat{n} \cdot \vec{\beta}_{-}}\right] .
\end{aligned}
$$

Comparing this with (2.1) we see that the soft graviton factor $\tilde{S}_{\mathrm{gr}}$, extracted from classical radiation, is given by

$$
\begin{aligned}
\tilde{S}_{\mathrm{gr}}(\varepsilon, k)= & -\omega^{-1} m \varepsilon^{i j}\left\{\frac{1}{1-\hat{n} \cdot \vec{\beta}_{+}} \frac{1}{\sqrt{1-\vec{\beta}_{+}^{2}}} \beta_{+i} \beta_{+j}-\frac{1}{1-\hat{n} \cdot \vec{\beta}_{-}} \frac{1}{\sqrt{1-\vec{\beta}_{-}^{2}}} \beta_{-i} \beta_{-j}\right\} \\
& -i m \varepsilon^{i j} \ln \omega^{-1}\left[\frac{1}{\sqrt{1-\vec{\beta}_{+}^{2}}} \beta_{+i} \beta_{+j} C_{+} \frac{1}{1-\hat{n} \cdot \vec{\beta}_{+}}-\frac{1}{\sqrt{1-\vec{\beta}_{-}^{2}}} \beta_{-i} \beta_{-j} C_{-} \frac{1}{1-\hat{n} \cdot \vec{\beta}_{-}}\right],
\end{aligned}
$$

for transverse polarization tensor $\varepsilon$. This agrees with $S_{\text {gr }}$ given in (2.15) upon replacing $\ln |t|$ by $\ln \omega^{-1}$.

\subsection{Gravitational radiation from scattering via gravitational interaction}

We shall now consider the scattering of a probe of mass $m$ by a massive scatterer of mass $M_{0}$ due to gravitational interaction. We shall assume that the impact parameter (the distance of closest approach) is large compared to the Schwarzschild radius of the scatterer and work to first order in the ratio of the Schwarzschild radius $M_{0} /(4 \pi)$ and the impact parameter. The radiative part of the gravitational field during such scattering was analyzed in [97]. After making appropriate changes in the signs and normalization factors described in [85], it is given by a sum of four terms:

$$
\tilde{e}_{i j}=\tilde{e}_{i j}^{(1)}+\tilde{e}_{i j}^{(2)}+\tilde{e}_{i j}^{(3)}+\tilde{e}_{i j}^{(4)} .
$$

$\tilde{e}^{(1)}$ is given by

$$
\tilde{e}_{i j}^{(1)}(\omega, \vec{x})=\frac{m e^{i \omega R}}{4 \pi R} \int \frac{d t}{1+2 \varphi(\vec{r}(t))} \frac{d t}{d \sigma} v_{i} v_{j} e^{i \omega(t-\hat{n} \cdot \vec{r}(t))}+\text { boundary terms },
$$


where $\vec{r}(t)$ denotes the trajectory of the particle,

$$
R \equiv|\vec{x}|, \quad \hat{n} \equiv \frac{\vec{x}}{|\vec{x}|},
$$

and $\varphi(\vec{r})$ is the gravitational potential:

$$
\varphi(\vec{r})=-\frac{M_{0}}{8 \pi|\vec{r}|},
$$

in the $8 \pi G=1$ unit. The other $\tilde{e}^{(i)}$ 's are given by

$$
\begin{aligned}
& \tilde{e}_{i j}^{(2)}(\omega, \vec{x})=i \frac{M_{0} m}{32 \pi^{2} \omega} \frac{e^{i \omega R}}{R} \int d t \frac{d t}{d \sigma}\left(1+\vec{v}^{2}\right)\left(\partial_{i}^{\prime} \partial_{j}^{\prime}-\frac{1}{2} \delta_{i j} \partial_{k}^{\prime} \partial_{k}^{\prime}\right)\left\{\ln \frac{\left|\vec{r}^{\prime}\right|+\hat{n} \cdot \vec{r}^{\prime}}{R} e^{i \omega\left(t-\hat{n} \cdot \vec{r}^{\prime}\right)}\right. \\
& \left.+\int_{\left|\vec{r}^{\prime}\right|+\hat{n} . \vec{r}^{\prime}}^{\infty} \frac{d u}{u} e^{i \omega\left(t-\hat{n} . \vec{r}^{\prime}+u\right)}\right\}\left.\right|_{\vec{r}^{\prime}=\vec{r}(t)}, \quad \partial_{i}^{\prime} \equiv \frac{\partial}{\partial r^{\prime i}}, \\
& \tilde{e}_{i j}^{(3)}(\omega, \vec{x})=-i \frac{M_{0} m}{16 \pi^{2}} \omega \frac{e^{i \omega R}}{R} \int d t \frac{d t}{d \sigma} v_{i} v_{j}\left\{\ln \frac{|\vec{r}(t)|+\hat{n} \cdot \vec{r}(t)}{R} e^{i \omega(t-\hat{n} . \vec{r}(t))}\right. \\
& \left.+\int_{|\vec{r}(t)|+\hat{n} . \vec{r}(t)}^{\infty} \frac{d u}{u} e^{i \omega(t-\hat{n} . \vec{r}(t)+u)}\right\}
\end{aligned}
$$

and

$$
\begin{gathered}
\tilde{e}_{i j}^{(4)}(\omega, \vec{x})=-\frac{M_{0} m}{16 \pi^{2}} \frac{e^{i \omega R}}{R} \int d t \frac{d t}{d \sigma}\left(v_{i} \partial_{j}^{\prime}+v_{j} \partial_{i}^{\prime}\right)\left\{\ln \frac{\left|\vec{r}^{\prime}\right|+\hat{n} \cdot \vec{r}^{\prime}}{R} e^{i \omega\left(t-\hat{n} \cdot \vec{r}^{\prime}\right)}\right. \\
\left.+\int_{\left|\vec{r}^{\prime}\right|+\hat{n} \cdot \vec{r}^{\prime}}^{\infty} \frac{d u}{u} e^{i \omega\left(t-\hat{n} \cdot \vec{r}^{\prime}+u\right)}\right\}\left.\right|_{\vec{r}^{\prime}=\vec{r}(t)}
\end{gathered}
$$

where

$$
\begin{aligned}
\frac{d t}{d \sigma} & =\left\{\left(1-\frac{M_{0}}{4 \pi|\vec{r}(t)|}\right)-\left(1-\frac{M_{0}}{4 \pi|\vec{r}(t)|}\right)^{-1} \vec{v}(t)^{2}\right\}^{-1 / 2} \\
& \simeq \frac{1}{\sqrt{1-\vec{v}(t)^{2}}}\left\{1+\frac{M_{0}}{8 \pi|\vec{r}(t)|} \frac{1+\vec{v}(t)^{2}}{1-\vec{v}(t)^{2}}\right\} \text { for large }|\vec{r}(t)|
\end{aligned}
$$

We begin with the evaluation of $\tilde{e}_{i j}^{(1)}$. We have

$$
e^{i \omega(t-\hat{n} . \vec{r}(t))}=\frac{1}{i \omega} \frac{1}{1-\hat{n} . \vec{v}(t)} \frac{d}{d t} e^{i \omega(t-\hat{n} . \vec{r}(t))} .
$$

Substituting this into (5.26) and integrating by parts we get

$$
\tilde{e}_{i j}^{(1)}(\omega, \vec{x})=-\frac{m}{4 \pi R} e^{i \omega R} \frac{1}{i \omega} \int d t e^{i \omega(t-\hat{n} . \vec{r}(t))} \frac{d}{d t}\left[\frac{1}{1-\hat{n} . \vec{v}(t)} \frac{1}{1+2 \varphi(\vec{r}(t))} \frac{d t}{d \sigma} v_{i} v_{j}\right] .
$$

Parametrizing $\vec{r}(t)$ for large $|t|$ as in (4.5) and using (5.32) we get, as $t \rightarrow \pm \infty$,

$$
\begin{aligned}
& \frac{1}{1-\hat{n} \cdot \vec{v}(t)} \frac{1}{1+2 \varphi(\vec{r}(t))} \frac{d t}{d \sigma} v_{i} v_{j}=\frac{1}{1-\hat{n} \cdot \vec{v}(t)} \frac{1}{1-M_{0} /(4 \pi|\vec{r}(t)|)} \frac{d t}{d \sigma} v_{i} v_{j} \\
= & \frac{1}{1-\hat{n} \cdot \vec{\beta}_{ \pm}} \frac{1}{\sqrt{1-\vec{\beta}_{ \pm}^{2}}} \beta_{ \pm i} \beta_{ \pm j}\left[1-\frac{1}{t}\left\{C_{ \pm} \frac{1}{1-\hat{n} . \vec{\beta}_{ \pm}} \mp \frac{M_{0}}{8 \pi\left|\vec{\beta}_{ \pm}\right|} \frac{3-\vec{\beta}_{ \pm}^{2}}{1-\vec{\beta}_{ \pm}^{2}}+C_{ \pm} \frac{1}{1-\vec{\beta}_{ \pm}^{2}}\right\}\right], \\
& (t-\hat{n} \cdot \vec{r}(t))=t\left(1-\hat{n} . \vec{\beta}_{ \pm}\right)+C_{ \pm} \hat{n} \cdot \vec{\beta}_{ \pm} \ln |t| .
\end{aligned}
$$


Comparing (5.34) with (3.5) and (5.35) with (3.4) we see that (5.34) takes the form of the integral $I_{1}$ with

$$
\begin{aligned}
f_{ \pm}= & i \frac{m}{4 \pi R} e^{i \omega R} \frac{1}{1-\hat{n} \cdot \vec{\beta}_{ \pm}} \frac{1}{\sqrt{1-\vec{\beta}_{ \pm}^{2}}} \beta_{ \pm i} \beta_{ \pm j}, \\
k_{ \pm}= & -i \frac{m}{4 \pi R} e^{i \omega R} \frac{1}{1-\hat{n} \cdot \vec{\beta}_{ \pm}} \frac{1}{\sqrt{1-\vec{\beta}_{ \pm}^{2}}} \beta_{ \pm i} \beta_{ \pm j} \\
& \left\{C_{ \pm} \frac{1}{1-\hat{n} \cdot \vec{\beta}_{ \pm}} \mp \frac{M_{0}}{8 \pi\left|\vec{\beta}_{ \pm}\right|} \frac{3-\vec{\beta}_{ \pm}^{2}}{1-\vec{\beta}_{ \pm}^{2}}+C_{ \pm} \frac{1}{1-\vec{\beta}_{ \pm}^{2}}\right\}, \\
a_{ \pm}= & -\left(1-\hat{n} \cdot \vec{\beta}_{ \pm}\right) .
\end{aligned}
$$

Therefore (3.5) gives

$$
\begin{aligned}
\tilde{e}_{i j}^{(1)}= & i \omega^{-1} \frac{m}{4 \pi R} e^{i \omega R}\left\{\frac{1}{1-\hat{n} \cdot \vec{\beta}_{+}} \frac{1}{\sqrt{1-\vec{\beta}_{+}^{2}}} \beta_{+i} \beta_{+j}-\frac{1}{1-\hat{n} \cdot \vec{\beta}_{-}} \frac{1}{\sqrt{1-\vec{\beta}_{-}^{2}}} \beta_{-i} \beta_{-j}\right\} \\
& -\frac{m}{4 \pi R} e^{i \omega R} \ln \omega^{-1}\left[\frac{1}{\sqrt{1-\vec{\beta}_{+}^{2}}} \beta_{+i} \beta_{+j}\left\{C_{+} \frac{1}{1-\vec{n}_{+}}-\frac{M_{0}}{8 \pi\left|\vec{\beta}_{+}\right|} \frac{3-\vec{\beta}_{+}^{2}}{1-\vec{\beta}_{+}^{2}}+C_{+} \frac{1}{1-\vec{\beta}_{+}^{2}}\right\}\right. \\
& \left.-\frac{1}{\sqrt{1-\vec{\beta}_{-}^{2}}} \beta_{-i} \beta_{-j}\left\{C_{-} \frac{1}{1-\hat{n} \cdot \vec{\beta}_{-}}+\frac{M_{0}}{8 \pi\left|\vec{\beta}_{-}\right|} \frac{3-\vec{\beta}_{-}^{2}}{1-\vec{\beta}_{-}^{2}}+C_{-} \frac{1}{1-\vec{\beta}_{-}^{2}}\right\}\right] .
\end{aligned}
$$

Next we turn to $\tilde{e}_{i j}^{(3)}$ given in (5.30). Using (5.33) and doing an integration by parts, we can express $\tilde{e}_{i j}^{(3)}$ as

$$
\begin{aligned}
\tilde{e}_{i j}^{(3)}(\omega, \vec{x})=\frac{M_{0} m}{16 \pi^{2}} \frac{e^{i \omega R}}{R} \int d t e^{i \omega(t-\hat{n} \cdot \vec{r}(t))} \frac{d}{d t}\left[\frac{1}{1-\hat{n} \cdot \vec{v}(t)} \frac{d t}{d \sigma} v_{i} v_{j}\right. \\
\left.\left\{\ln (|\vec{r}(t)|+\hat{n} \cdot \vec{r}(t))+\int_{|\vec{r}(t)|+\hat{n} \cdot \vec{r}(t)}^{\infty} \frac{d u}{u} e^{i \omega u}\right\}\right] .
\end{aligned}
$$

This integral is of the form $I_{2}$ given in (3.5) and therefore gives the result: ${ }^{2}$

$$
\tilde{e}_{i j}^{(3)}=-\frac{M_{0} m}{16 \pi^{2}} \ln (\omega R) \frac{e^{i \omega R}}{R}\left\{\frac{1}{1-\hat{n} \cdot \vec{\beta}_{+}} \frac{1}{\sqrt{1-\vec{\beta}_{+}^{2}}} \beta_{+i} \beta_{+j}-\frac{1}{1-\hat{n} \cdot \vec{\beta}_{-}} \frac{1}{\sqrt{1-\vec{\beta}_{-}^{2}}} \beta_{-i} \beta_{-j}\right\} \text {. }
$$

This term can be understood as arising from multiplication of the first line of (5.37) by the phase factor $\exp \left[i \omega M_{0} \ln (\omega R) /(4 \pi)\right]$. This is precisely the additional phase factor (1.6) arising due to gravitational drag and backscattering experienced by the emitted radiation due to the gravitational field of the mass $M_{0}$.

\footnotetext{
${ }^{2}$ This term was ignored in [97] since at large impact parameter $\vec{\beta}_{+} \simeq \vec{\beta}_{-}$, and the term inside the curly bracket of (5.39) is small. However we can easily conceive a slightly different situation where a pair of particles undergo an elastic collision in the black hole background, causing a change of order unity in each of their velocities. In this case $\vec{\beta}_{+}-\vec{\beta}_{-}$will be of order unity for each of these particles. The gravitational field produced during this process will be given by the sum of the contributions due to these two particles, each of which can be evaluated using the result given in this section.
} 
Next we consider $\tilde{e}_{i j}^{(4)}$ given in (5.31). It can be expressed as

$$
\begin{aligned}
\tilde{e}_{i j}^{(4)}(\omega, \vec{x})= & -\frac{M_{0} m}{16 \pi^{2}} \frac{e^{i \omega R}}{R} \int d t \frac{d t}{d \sigma} e^{i \omega(t-\hat{n} \cdot \vec{r}(t))} \\
& {\left[(-i \omega)\left(v_{i} n_{j}+v_{j} n_{i}\right)\left\{\ln \left(\left|\vec{r}^{\prime}\right|+\hat{n} \cdot \vec{r}^{\prime}\right)+\int_{\left|\vec{r}^{\prime}\right|+\hat{n} \cdot \vec{r}^{\prime}}^{\infty} \frac{d u}{u} e^{i \omega u}\right\}\right.} \\
& \left.+\frac{1}{\left|\vec{r}^{\prime}\right|+\hat{n} \cdot \vec{r}^{\prime}}\left\{v_{i}\left(\frac{r_{j}^{\prime}}{\left|\vec{r}^{\prime}\right|}+\hat{n}_{j}\right)+v_{j}\left(\frac{r_{i}^{\prime}}{\left|\vec{r}^{\prime}\right|}+\hat{n}_{i}\right)\right\}\left\{1-e^{i \omega\left(\left|\vec{r}^{\prime}\right|+\hat{n} \cdot \vec{r}^{\prime}\right)}\right\}\right]\left.\right|_{\vec{r}^{\prime}=\vec{r}(t)} .
\end{aligned}
$$

The contribution to the integral from the term in the second line vanishes after $\tilde{e}_{i j}^{(4)}$ is contracted with the polarization tensor $\varepsilon^{i j}$, since $\varepsilon_{i j} \hat{n}^{j}=\varepsilon_{i j} k^{j} /|\vec{k}|=0$. The contribution from the last line has the same structure as $I_{3}$ and therefore also does not generate any term proportional to $\omega^{-1}$ or $\ln \omega^{-1}$.

We now turn to the computation of $\tilde{e}_{i j}^{(2)}$ given in (5.29). With the gauge condition $\varepsilon^{i}{ }_{i}=0$ given in $(2.8)$, the term proportional to $\delta_{i j}$ does not contribute to $\varepsilon^{i j} \tilde{e}_{i j}$. Now we have

$$
\begin{aligned}
& \partial_{j}^{\prime}\left\{\ln \left(\left|\vec{r}^{\prime}\right|+\hat{n} \cdot \vec{r}^{\prime}\right) e^{i \omega\left(t-\hat{n} \cdot \vec{r}^{\prime}\right)}+\int_{\left|\vec{r}^{\prime}\right|+\hat{n} \cdot \vec{r}^{\prime}}^{\infty} \frac{d u}{u} e^{i \omega\left(t-\hat{n} \cdot \vec{r}^{\prime}+u\right)}\right\} \\
= & -i \omega \hat{n}_{j}\left\{\ln \left(\left|\vec{r}^{\prime}\right|+\hat{n} \cdot \vec{r}^{\prime}\right) e^{i \omega\left(t-\hat{n} \cdot \vec{r}^{\prime}\right)}+\int_{\left|\vec{r}^{\prime}\right|+\hat{n} \cdot \vec{r}^{\prime}}^{\infty} \frac{d u}{u} e^{i \omega\left(t-\hat{n} \cdot \vec{r}^{\prime}+u\right)}\right\} \\
& +\frac{1}{\left|\vec{r}^{\prime}\right|+\hat{n} \cdot \vec{r}^{\prime}}\left(\frac{r_{j}^{\prime}}{\left|\vec{r}^{\prime}\right|}+\hat{n}_{j}\right)\left\{e^{i \omega\left(t-\hat{n} \cdot \vec{r}^{\prime}\right)}-e^{i \omega\left(t+\left|\vec{r}^{\prime}\right|\right)}\right\}
\end{aligned}
$$

Substituting this into (5.29) we see that the contribution from the term in the first line of the right hand side of (5.41) will vanish after contraction with $\varepsilon^{i j}$. This allows us to focus on the term in the last line of (5.41). Now we have

$$
\begin{aligned}
& \partial_{i}^{\prime}\left[\frac{1}{\left|\vec{r}^{\prime}\right|+\hat{n} \cdot \vec{r}^{\prime}}\left(\frac{r_{j}^{\prime}}{\left|\vec{r}^{\prime}\right|}+\hat{n}_{j}\right)\left\{e^{i \omega\left(t-\hat{n} \cdot \vec{r}^{\prime}\right)}-e^{i \omega\left(t+\left|\vec{r}^{\prime}\right|\right)}\right\}\right] \\
= & -\frac{1}{\left(\left|\vec{r}^{\prime}\right|+\hat{n} \cdot \vec{r}^{\prime}\right)^{2}}\left(\frac{r_{i}^{\prime}}{\left|\vec{r}^{\prime}\right|}+\hat{n}_{i}\right)\left(\frac{r_{j}^{\prime}}{\left|\vec{r}^{\prime}\right|}+\hat{n}_{j}\right)\left\{e^{i \omega\left(t-\hat{n} \cdot \vec{r}^{\prime}\right)}-e^{i \omega\left(t+\left|\vec{r}^{\prime}\right|\right)}\right\} \\
& +\frac{1}{\left|\vec{r}^{\prime}\right|+\hat{n} \cdot \vec{r}^{\prime}}\left\{\frac{\delta_{i j}}{\left|\vec{r}^{\prime}\right|}-\frac{r_{i}^{\prime} r_{j}^{\prime}}{\left(\left|\vec{r}^{\prime}\right|\right)^{3}}\right\}\left\{e^{i \omega\left(t-\hat{n} \cdot \vec{r}^{\prime}\right)}-e^{i \omega\left(t+\left|\vec{r}^{\prime}\right|\right)}\right\} \\
& -i \omega \frac{1}{\left|\vec{r}^{\prime}\right|+\hat{n} \cdot \vec{r}^{\prime}}\left(\frac{r_{j}^{\prime}}{\left|\vec{r}^{\prime}\right|}+\hat{n}_{j}\right)\left\{e^{i \omega\left(t-\hat{n} \cdot \vec{r}^{\prime}\right)} \hat{n}_{i}+e^{i \omega\left(t+\left|\vec{r}^{\prime}\right|\right)} \frac{r_{i}^{\prime}}{\left|\vec{r}^{\prime}\right|}\right\} .
\end{aligned}
$$

Before substituting this into (5.29) we note that the term proportional to $\delta_{i j}$ does not contribute to $\varepsilon^{i j} \tilde{e}_{i j}$ due to the $\varepsilon^{i}{ }_{i}=0$ condition. Also the terms proportional to $\hat{n}_{i}$ and $\hat{n}_{j}$ can be dropped since $\hat{n}_{i}=k_{i} /|\vec{k}|$ and we have the $k_{i} \varepsilon^{i j}=0$ condition in (2.8). Substituting 
this into (5.29) we see that the relevant part of $\tilde{e}_{i j}^{(2)}$ is given by

$$
\begin{aligned}
\tilde{e}_{i j}^{(2)}(\omega, \vec{x})= & i \frac{M_{0} m}{32 \pi^{2} \omega} \frac{e^{i \omega R}}{R} \int d t \frac{d t}{d \sigma}\left(1+\vec{v}^{2}\right) \\
& {\left[-\frac{1}{\left(\left|\vec{r}^{\prime}\right|+\hat{n} \cdot \vec{r}^{\prime}\right)^{2}} \frac{r_{i}^{\prime} r_{j}^{\prime}}{\left(\left|\vec{r}^{\prime}\right|\right)^{2}}\left\{e^{i \omega\left(t-\hat{n} . \vec{r}^{\prime}\right)}-e^{i \omega\left(t+\left|\vec{r}^{\prime}\right|\right)}\right\}\right.} \\
& -\frac{1}{\left|\vec{r}^{\prime}\right|\left(\left|\vec{r}^{\prime}\right|+\hat{n} . \vec{r}^{\prime}\right)} \frac{r_{i}^{\prime} r_{j}^{\prime}}{\left(\left|\vec{r}^{\prime}\right|\right)^{2}}\left\{e^{i \omega\left(t-\hat{n} \cdot \vec{r}^{\prime}\right)}-e^{i \omega\left(t+\left|\vec{r}^{\prime}\right|\right)}\right\} \\
& \left.-i \omega \frac{1}{\left|\vec{r}^{\prime}\right|+\hat{n} . \vec{r}^{\prime}} \frac{r_{i}^{\prime} r_{j}^{\prime}}{\left(\left|\vec{r}^{\prime}\right|\right)^{2}} e^{i \omega\left(t+\left|\vec{r}^{\prime}\right|\right)}\right]_{\vec{r}^{\prime}=\vec{r}(t)} .
\end{aligned}
$$

Using the asymptotic behavior (4.5) we see that the contribution from the second and third line have the form $I_{4}$ and the contribution from the last line has the form $I_{5}$, both given in (3.5). The result is

$$
\begin{aligned}
& \tilde{e}_{i j}^{(2)}(\omega, \vec{x})=\ln \omega^{-1} \frac{M_{0} m}{32 \pi^{2}} \frac{e^{i \omega R}}{R}[- \frac{\left(1+\vec{\beta}^{2}\right) \beta_{i} \beta_{j}}{\vec{\beta}^{2} \sqrt{1-\vec{\beta}^{2}}}\left\{\frac{1}{(\epsilon|\vec{\beta}|+\hat{n} \cdot \vec{\beta})^{2}}+\frac{1}{(\epsilon|\vec{\beta}|+\hat{n} \cdot \vec{\beta}) \epsilon|\vec{\beta}|}\right\}(\epsilon|\vec{\beta}|+\hat{n} \cdot \vec{\beta}) \\
&\left.+\frac{\left(1+\vec{\beta}^{2}\right) \beta_{i} \beta_{j}}{\vec{\beta}^{2} \sqrt{1-\vec{\beta}^{2}}} \frac{1}{(\epsilon|\vec{\beta}|+\hat{n} \cdot \vec{\beta})}\right]_{-}^{+} \\
&=-\ln \omega^{-1} \frac{M_{0} m}{32 \pi^{2}} \frac{e^{i \omega R}}{R}\left[\frac{\left(1+\vec{\beta}^{2}\right) \beta_{i} \beta_{j}}{\epsilon|\vec{\beta}|^{3} \sqrt{1-\vec{\beta}^{2}}}\right]_{-}^{+}
\end{aligned}
$$

where $\epsilon$ is +1 for outgoing states and -1 for ingoing states. This gives

$$
\tilde{e}_{i j}^{(2)}(\omega, \vec{x})=-\ln \omega^{-1} \frac{M_{0} m}{32 \pi^{2}} \frac{e^{i \omega R}}{R}\left[\frac{\left(1+\vec{\beta}_{+}^{2}\right) \beta_{+i} \beta_{+j}}{\left|\vec{\beta}_{+}\right|^{3} \sqrt{1-\vec{\beta}_{+}^{2}}}+\frac{\left(1+\vec{\beta}_{-}^{2}\right) \beta_{-i} \beta_{-j}}{\left|\vec{\beta}_{-}\right|^{3} \sqrt{1-\vec{\beta}_{-}^{2}}}\right] .
$$

Adding (5.37), (5.39) and (5.45), using (5.25) and comparing the result with (2.1) with the extra phase factor (1.6) on the right hand side (which cancels (5.39)), we get the following prediction for the soft factor from the classical scattering results:

$$
\begin{aligned}
\tilde{S}_{\mathrm{gr}}= & -m \omega^{-1} \varepsilon^{i j}\left\{\frac{1}{1-\hat{n} \cdot \vec{\beta}_{+}} \frac{1}{\sqrt{1-\vec{\beta}_{+}^{2}}} \beta_{+i} \beta_{+j}-\frac{1}{1-\hat{n} \cdot \vec{\beta}_{-}} \frac{1}{\sqrt{1-\vec{\beta}_{-}^{2}}} \beta_{-i} \beta_{-j}\right\} \\
-i m \ln \omega^{-1} \varepsilon^{i j} & {\left[\frac{1}{\sqrt{1-\vec{\beta}_{+}^{2}}} \beta_{+i} \beta_{+j}\left\{C_{+} \frac{1}{1-\hat{n} \cdot \vec{\beta}_{+}}-\frac{M_{0}}{8 \pi\left|\vec{\beta}_{+}\right|^{3}} \frac{3 \vec{\beta}_{+}^{2}-1}{1-\vec{\beta}_{+}^{2}}+C_{+} \frac{1}{1-\vec{\beta}_{+}^{2}}\right\}\right.} \\
& \left.-\frac{1}{\sqrt{1-\vec{\beta}_{-}^{2}}} \beta_{-i} \beta_{-j}\left\{C_{-} \frac{1}{1-\hat{n} \cdot \vec{\beta}_{-}}+\frac{M_{0}}{8 \pi\left|\vec{\beta}_{-}\right|} \frac{3 \vec{\beta}_{-}^{2}-1}{1-\vec{\beta}_{-}^{2}}+C_{-} \frac{1}{1-\vec{\beta}_{-}^{2}}\right\}\right] .
\end{aligned}
$$


In order to compare this to $(2.15)$ we need to find the relation between $M_{0}$ and $C_{ \pm}$. Let $\vec{v}(t)$ be the velocity of the particle at large $|t|$ when the particle is at a distance $r$ from the black hole and $\vec{\beta}$ be the velocity as $|t| \rightarrow \infty$. The expression of the total energy of the particle in the $8 \pi G=1$ units is given by

$$
E=m\left(1-\frac{M_{0}}{4 \pi r}\right)\left\{\left(1-\frac{M_{0}}{4 \pi r}\right)-\left(1-\frac{M_{0}}{4 \pi r}\right)^{-1} \vec{v}^{2}\right\}^{-1 / 2},
$$

so that the conservation of energy gives

$$
\left(1-\frac{M_{0}}{4 \pi r}\right)\left\{\left(1-\frac{M_{0}}{4 \pi r}\right)-\left(1-\frac{M_{0}}{4 \pi r}\right)^{-1} \vec{v}^{2}\right\}^{-1 / 2}=\left(1-\vec{\beta}^{2}\right)^{-1 / 2} .
$$

To first order in an expansion in powers of $M_{0}$ this gives

$$
\vec{v}(t)=\vec{\beta}\left(1+\frac{M_{0}}{8 \pi \vec{\beta}^{2} r}\left(1-3 \vec{\beta}^{2}\right)\right)=\vec{\beta}\left(1+\frac{M_{0}}{8 \pi|\vec{\beta}|^{3}|t|}\left(1-3 \vec{\beta}^{2}\right)\right)
$$

where we have used $r=|\vec{\beta}||t|$. Comparing this with (4.5) we get

$$
C_{ \pm}=\mp \frac{M_{0}\left(1-3 \vec{\beta}_{ \pm}^{2}\right)}{8 \pi\left|\vec{\beta}_{ \pm}\right|^{3}} \text {. }
$$

Using this we can express (5.46) as

$$
\begin{aligned}
\tilde{S}_{\mathrm{gr}}= & -m \omega^{-1} \varepsilon^{i j}\left\{\frac{1}{1-\hat{n} \cdot \vec{\beta}_{+}} \frac{1}{\sqrt{1-\vec{\beta}_{+}^{2}}} \beta_{+i} \beta_{+j}-\frac{1}{1-\hat{n} \cdot \vec{\beta}_{-}} \frac{1}{\sqrt{1-\vec{\beta}_{-}^{2}}} \beta_{-i} \beta_{-j}\right\} \\
& -i m \varepsilon^{i j} \ln \omega^{-1}\left[\frac{1}{\sqrt{1-\vec{\beta}_{+}^{2}}} \beta_{+i} \beta_{+j} C_{+} \frac{1}{1-\hat{n} \cdot \vec{\beta}_{+}}-\frac{1}{\sqrt{1-\vec{\beta}_{-}^{2}}} \beta_{-i} \beta_{-j} C_{-} \frac{1}{1-\hat{n} \cdot \vec{\beta}_{-}}\right] .
\end{aligned}
$$

This agrees with (2.15) with $\ln |t|$ replaced by $\ln \omega^{-1}$.

\section{Acknowledgments}

We wish to thank Miguel Campiglia, Walter Golberger, Ira Rothstein and Biswajit Sahoo for discussions. Work of A.L. is supported in part by Ramanujan Fellowship. The work of A.S. was supported in part by the J.C. Bose fellowship of the Department of Science and Technology, India.

\section{A Evaluation of some integrals}

Our goal in this appendix will be to compute the $1 / \omega$ and $\ln \omega$ terms in the following integrals in $\omega \rightarrow 0$ limit.

$$
\begin{aligned}
& I_{1} \equiv \frac{1}{\omega} \int_{-\infty}^{\infty} d t e^{-i \omega g(t)} f^{\prime}(t), \\
& I_{2} \equiv \int_{-\infty}^{\infty} d t e^{-i \omega g(t)} \frac{d}{d t}\left[f(t)\left\{\ln \frac{h(t)}{R}+\int_{h(t)}^{\infty} e^{i \omega u} \frac{d u}{u}\right\}\right],
\end{aligned}
$$




$$
\begin{aligned}
I_{3} & \equiv \int_{-\infty}^{\infty} d t \frac{1}{r(t)} f(t)\left[e^{-i \omega g(t)}-e^{-i \omega h(t)}\right], \\
I_{4} & \equiv \frac{1}{\omega} \int_{-\infty}^{\infty} d t \frac{1}{r(t)^{2}} f(t)\left[e^{-i \omega g(t)}-e^{-i \omega h(t)}\right], \\
I_{5} & =\int_{-\infty}^{\infty} d t \frac{1}{r(t)} f(t) e^{-i \omega g(t)},
\end{aligned}
$$

where, as described in (3.4), $f(t), g(t), h(t)$ are smooth functions with the property

$$
\begin{array}{lll}
f(t)=f_{ \pm}+\frac{k_{ \pm}}{t}, & g(t) \rightarrow a_{ \pm} t+b_{ \pm} \ln |t|, & \\
h(t) \rightarrow p_{ \pm} t+q_{ \pm} \ln |t|, & r(t) \rightarrow c_{ \pm} t+d_{ \pm} \ln |t|, \quad \text { as } \quad t \rightarrow \pm \infty .
\end{array}
$$

We shall evaluate the integrals by separately estimating their contributions from the four regions: $|t| \sim 1,1 \ll\left|t \ll \omega^{-1},\right| t \mid \sim \omega^{-1}$ and $|t| \gg \omega^{-1}$.

\section{A.1 Evaluation of $I_{1}$}

We express $I_{1}$ as

$$
I_{1}=\frac{1}{\omega} \int_{-\infty}^{\infty} d t f^{\prime}(t)+\frac{1}{\omega} \int_{-\infty}^{\infty} d t\left\{e^{-i \omega g(t)}-1\right\} f^{\prime}(t)
$$

The first term gives $\omega^{-1}\left(f_{+}-f_{-}\right)$. The second term can be evaluated by dividing the integration region into different segments. In the region $t \sim 1$ the term inside the curly bracket is of order $\omega$ and we get a finite contribution. In the region $1 \ll|t| \ll \omega^{-1}$ we can approximate the integral as

$$
-i \int_{1 \ll|t| \ll \omega^{-1}} d t g(t) f^{\prime}(t) \simeq i \int_{1 \ll|t| \ll \omega^{-1}} d t \frac{a_{ \pm} k_{ \pm}}{t} \simeq i\left(a_{+} k_{+}-a_{-} k_{-}\right) \ln \omega^{-1} .
$$

The last step can be justified as follows. Let us fix the integration range to be $\left[a, b \omega^{-1}\right]$ where $a$ and $b$ some fixed numbers with $a \gg 1$ and $b \ll 1$. The right hand side of the above equation can then be approximated as

$$
i\left(a_{+} k_{+}-a_{-} k_{-}\right)\left[\ln \omega^{-1}+\ln \frac{b}{a}\right] .
$$

Even though $\frac{b}{a} \ll 1$, as $\omega^{-1}$ becomes large, we can ignore $\ln \frac{b}{a}$ compared to $\ln \omega^{-1}$, arriving at the right hand side of (A.8).

In the region $|t| \sim \omega^{-1}$ and $|t|>\omega^{-1}$ the magnitude of the integral is bounded by a term of order

$$
\omega^{-1} \int_{|t|>\omega^{-1}} 2\left\{\left|k_{ \pm}\right| t^{-2}\right\} d t \sim 2\left|k_{ \pm}\right|
$$

Therefore for small $\omega, I_{1}$ can be estimated to be

$$
I_{1}=\omega^{-1}\left(f_{+}-f_{-}\right)+i\left(a_{+} k_{+}-a_{-} k_{-}\right) \ln \omega^{-1}+\text { finite } .
$$




\section{A.2 Evaluation of $I_{2}$}

Let us express $I_{2}$ as

$$
I_{2} \equiv \int_{-\infty}^{\infty} d t e^{-i \omega g(t)}\left[f^{\prime}(t)\left\{\ln \frac{h(t)}{R}+\int_{h(t)}^{\infty} e^{i \omega u} \frac{d u}{u}\right\}+f(t) h^{\prime}(t) h(t)^{-1}\left(1-e^{i \omega h(t)}\right)\right] .
$$

While integrating over the region $|t| \sim 1$, we can replace $e^{i \omega t}$ by 1 . Also in this region the integral inside the curly bracket can be evaluated by changing variable from $u$ to $v=\omega u$, and yields $\ln \omega^{-1}$ plus a finite term. Therefore the term inside the curly bracket is given by $-\ln (R \omega)$ plus a finite term, and the integration over $t$ produces a term

$$
-\ln (R \omega)\left(f_{+}-f_{-}\right)+\text {finite } .
$$

For $1 \ll|t| \ll \omega^{-1}$ the term with the $f^{\prime}(t)$ factor is of order $t^{-2} \times$ logarithmic terms and produces a finite result. On the other hand the $f(t) h^{\prime}(t)(h(t))^{-1}\left(1-e^{i \omega h(t)}\right)$ factor is of order $-i \omega f_{ \pm} p_{ \pm}$and gives negligible contribution to the integral from the $1 \ll|t| \ll \omega^{-1}$ region. For $|t| \sim \omega^{-1}$ the integrand is of order $t^{-1}$ and therefore gives a finite contribution to the integral. Finally for $t \gg \omega^{-1}$ the term proportional to $f^{\prime}(t)$ falls off as $t^{-2} \times$ logarithmic terms and its contribution to the integral is vanishes in the $\omega \rightarrow 0$ limit. In this range the term proportional to $f(t) h^{\prime}(t)(h(t))^{-1}$ may be approximated as

$$
\int_{\omega^{-1}}^{\infty} d t f_{ \pm} t^{-1}\left\{e^{-i a_{ \pm} \omega t}-e^{i\left(1-a_{ \pm}\right) \omega t}\right\}
$$

After changing variable to $u=-a_{ \pm} \omega t$ in the first term and $\left(1-a_{ \pm}\right) \omega t$ in the second term, each of the integrals can be converted to the form

$$
f_{ \pm} \int^{\infty} d u u^{-1} e^{i u}
$$

with finite lower limit of order unity. This gives a finite result. Therefore we get

$$
I_{2}=-\ln (R \omega)\left(f_{+}-f_{-}\right)+\text {finite } .
$$

A.3 Evaluation of $I_{3} \equiv \int_{-\infty}^{\infty} d t(r(t))^{-1} f(t)\left[e^{-i \omega g(t)}-e^{-i \omega h(t)}\right]$

The region $|t| \sim 1$ gives a finite contribution. For $1 \ll|t| \ll \omega^{-1}$ the integrand may be approximated as

$$
\left(c_{ \pm}\right)^{-1} f_{ \pm}(-i \omega)\left(a_{ \pm}-p_{ \pm}\right),
$$

and the integral receives negligible contribution from this region.

For $|t| \sim \omega^{-1}$ the term in the square bracket is of order unity. But the rest of the integrand is of order $\left(c_{ \pm} t\right)^{-1} f_{ \pm}$and integration over $t$ in the range $|t| \sim \omega^{-1}$ produces at most a term of order unity - there is no contribution proportional to $\ln \omega$. Finally for $|t| \gg \omega^{-1}$ the integrand has the same form as (A.14) with $\left(1-a_{ \pm}\right)$replaced by $p_{ \pm}$ in the second exponent, and an overall multiplicative factor $\left(c_{ \pm}\right)^{-1}$. Therefore it gives a finite result. 


\section{A.4 Evaluation of $I_{4} \equiv \omega^{-1} \int_{-\infty}^{\infty} d t(r(t))^{-2} f(t)\left[e^{-i \omega g(t)}-e^{-i \omega h(t)}\right]$}

We use

$$
\frac{1}{r(t)^{2}}=-\frac{d}{d t}\left\{\frac{1}{r(t)}\right\} \frac{1}{r^{\prime}(t)}
$$

Substituting this into the expression for $I_{4}$ and doing an integration by parts we get the following form of the integral for large $|t|$ :

$$
\frac{1}{\omega} \int d t \frac{1}{r(t)} \frac{d}{d t}\left(\frac{f(t)}{r^{\prime}(t)}\right)\left[e^{-i \omega g(t)}-e^{-i \omega h(t)}\right]-i \int d t \frac{f(t)}{r(t) r^{\prime}(t)}\left[g^{\prime}(t) e^{-i \omega g(t)}-h^{\prime}(t) e^{-i \omega h(t)}\right] .
$$

For $|t| \sim 1$ the integrands in both terms are finite in the $\omega \rightarrow 0$ limit and we get finite contribution to the integral. Using (A.6) we see that in the first term, part of the integrand outside the square bracket falls off as $1 /|t|^{3}$ for $|t| \gg 1$. On the other hand, using the inequality $|\sin u| \leq|u|$, we can see that the terms inside the square bracket of the first term is bounded by $\omega|g(t)-h(t)| \sim \omega|t|\left|a_{ \pm}-p_{ \pm}\right|$. Therefore integration over the region $|t|>1$ yields a finite result as $\omega \rightarrow 0$ for the first term.

The contribution from the second term can be evaluated by noting that for large $|t|$, $f(t) /\left\{r(t) r^{\prime}(t)\right\} \rightarrow f_{ \pm} c_{ \pm}^{-2} t^{-1}, g^{\prime}(t) \rightarrow a_{ \pm}$and $h^{\prime}(t) \rightarrow p_{ \pm}$. Therefore in the $1 \ll|t| \ll \omega^{-1}$ region the integrand behaves as $-i f_{ \pm} c_{ \pm}^{-2} t^{-1}\left(a_{ \pm}-p_{ \pm}\right)$and the dominant contribution to the integral is given by

$$
-i\left\{f_{+} c_{+}^{-2}\left(a_{+}-p_{+}\right)-f_{-} c_{-}^{-2}\left(a_{-}-p_{-}\right)\right\} \ln \omega^{-1} .
$$

For $|t| \sim \omega^{-1}$ the integrand is of order $t^{-1}$, producing a finite result for the integral. Finally for $|t| \gg \omega^{-1}$ the integrand is proportional to $t^{-1}\left[g^{\prime}(t) e^{-i \omega g(t)}-h^{\prime}(t) e^{-i \omega h(t)}\right]$. Each of these produces a finite contribution in the $\omega \rightarrow 0$ limit.

Therefore we get

$$
I_{4} \simeq-i\left\{f_{+} c_{+}^{-2}\left(a_{+}-p_{+}\right)-f_{-} c_{-}^{-2}\left(a_{-}-p_{-}\right)\right\} \ln \omega^{-1} .
$$

\section{A.5 Evaluation of $I_{5} \equiv \int_{-\infty}^{\infty} d t(r(t))^{-1} f(t) e^{-i \omega g(t)}$}

The $|t| \sim 1$ and $|t| \sim \omega^{-1}$ regions give finite contributions. The region $1 \ll|t| \ll \omega^{-1}$ gives

$$
\int_{1 \ll|t| \ll \omega^{-1}} d t \frac{f_{ \pm}}{c_{ \pm} t} \simeq \pm \frac{f_{ \pm}}{c_{ \pm}} \ln \omega^{-1}
$$

Finally in the region $|t| \gg \omega^{-1}$ the integral takes the form

$$
\int_{|t| \gg \omega^{-1}} d t \frac{f_{ \pm}}{c_{ \pm} t} e^{-i a_{ \pm} \omega t-i b_{ \pm} \omega \ln |t|}
$$

This is bounded by a finite number. Therefore the net contribution to $I_{5}$ is given by

$$
I_{5} \simeq\left(f_{+} c_{+}^{-1}-f_{-} c_{-}^{-1}\right) \ln \omega^{-1}
$$

Note that the result for $I_{3}$ follows from this. 
Open Access. This article is distributed under the terms of the Creative Commons Attribution License (CC-BY 4.0), which permits any use, distribution and reproduction in any medium, provided the original author(s) and source are credited.

\section{References}

[1] M. Gell-Mann and M.L. Goldberger, Scattering of low-energy photons by particles of spin 1/2, Phys. Rev. 96 (1954) 1433 [inSPIRE].

[2] F.E. Low, Bremsstrahlung of very low-energy quanta in elementary particle collisions, Phys. Rev. 110 (1958) 974 [INSPIRE].

[3] S. Saito, Low-energy theorem for Compton scattering, Phys. Rev. 184 (1969) 1894 [inSPIRE].

[4] T.H. Burnett and N.M. Kroll, Extension of the low soft photon theorem, Phys. Rev. Lett. 20 (1968) 86 [INSPIRE].

[5] J.S. Bell and R. Van Royen, On the low-burnett-kroll theorem for soft-photon emission, Nuovo Cim. A 60 (1969) 62 [inSPIRE].

[6] V. Del Duca, High-energy Bremsstrahlung Theorems for Soft Photons, Nucl. Phys. B 345 (1990) 369 [INSPIRE].

[7] S. Weinberg, Photons and Gravitons in s Matrix Theory: Derivation of Charge Conservation and Equality of Gravitational and Inertial Mass, Phys. Rev. 135 (1964) B1049 [INSPIRE].

[8] S. Weinberg, Infrared photons and gravitons, Phys. Rev. 140 (1965) B516 [INSPIRE].

[9] D.J. Gross and R. Jackiw, Low-Energy Theorem for Graviton Scattering, Phys. Rev. 166 (1968) 1287 [INSPIRE].

[10] R. Jackiw, Low-Energy Theorems for Massless Bosons: Photons and Gravitons, Phys. Rev. 168 (1968) 1623 [INSPIRE].

[11] M. Ademollo et al., Soft Dilations and Scale Renormalization in Dual Theories, Nucl. Phys. B 94 (1975) 221 [INSPIRE].

[12] J.A. Shapiro, On the Renormalization of Dual Models, Phys. Rev. D 11 (1975) 2937 [INSPIRE].

[13] C.D. White, Factorization Properties of Soft Graviton Amplitudes, JHEP 05 (2011) 060 [arXiv:1103.2981] [INSPIRE].

[14] F. Cachazo and A. Strominger, Evidence for a New Soft Graviton Theorem, arXiv: 1404.4091 [INSPIRE].

[15] E. Casali, Soft sub-leading divergences in Yang-Mills amplitudes, JHEP 08 (2014) 077 [arXiv: 1404.5551] [INSPIRE].

[16] B.U.W. Schwab and A. Volovich, Subleading Soft Theorem in Arbitrary Dimensions from Scattering Equations, Phys. Rev. Lett. 113 (2014) 101601 [arXiv:1404.7749] [INSPIRE].

[17] Z. Bern, S. Davies and J. Nohle, On Loop Corrections to Subleading Soft Behavior of Gluons and Gravitons, Phys. Rev. D 90 (2014) 085015 [arXiv:1405.1015] [INSPIRE].

[18] S. He, Y.-t. Huang and C. Wen, Loop Corrections to Soft Theorems in Gauge Theories and Gravity, JHEP 12 (2014) 115 [arXiv:1405.1410] [INSPIRE].

[19] A.J. Larkoski, Conformal Invariance of the Subleading Soft Theorem in Gauge Theory, Phys. Rev. D 90 (2014) 087701 [arXiv: 1405.2346] [INSPIRE].

[20] F. Cachazo and E.Y. Yuan, Are Soft Theorems Renormalized?, arXiv:1405.3413 [InSPIRE]. 
[21] N. Afkhami-Jeddi, Soft Graviton Theorem in Arbitrary Dimensions, arXiv:1405.3533 [INSPIRE].

[22] B.U.W. Schwab, Subleading Soft Factor for String Disk Amplitudes, JHEP 08 (2014) 062 [arXiv: 1406.4172] [INSPIRE].

[23] M. Bianchi, S. He, Y.-t. Huang and C. Wen, More on Soft Theorems: Trees, Loops and Strings, Phys. Rev. D 92 (2015) 065022 [arXiv:1406.5155] [INSPIRE].

[24] J. Broedel, M. de Leeuw, J. Plefka and M. Rosso, Constraining subleading soft gluon and graviton theorems, Phys. Rev. D 90 (2014) 065024 [arXiv:1406.6574] [InSPIRE].

[25] Z. Bern, S. Davies, P. Di Vecchia and J. Nohle, Low-Energy Behavior of Gluons and Gravitons from Gauge Invariance, Phys. Rev. D 90 (2014) 084035 [arXiv:1406.6987] [INSPIRE].

[26] C.D. White, Diagrammatic insights into next-to-soft corrections, Phys. Lett. B 737 (2014) 216 [arXiv: 1406.7184] [INSPIRE].

[27] M. Zlotnikov, Sub-sub-leading soft-graviton theorem in arbitrary dimension, JHEP 10 (2014) 148 [arXiv: 1407.5936] [INSPIRE].

[28] C. Kalousios and F. Rojas, Next to subleading soft-graviton theorem in arbitrary dimensions, JHEP 01 (2015) 107 [arXiv:1407.5982] [INSPIRE].

[29] Y.-J. Du, B. Feng, C.-H. Fu and Y. Wang, Note on Soft Graviton theorem by KLT Relation, JHEP 11 (2014) 090 [arXiv: 1408.4179] [INSPIRE].

[30] D. Bonocore, E. Laenen, L. Magnea, L. Vernazza and C.D. White, The method of regions and next-to-soft corrections in Drell-Yan production, Phys. Lett. B 742 (2015) 375 [arXiv: 1410.6406] [INSPIRE].

[31] B.U.W. Schwab, A Note on Soft Factors for Closed String Scattering, JHEP 03 (2015) 140 [arXiv:1411.6661] [INSPIRE].

[32] A. Sabio Vera and M.A. Vazquez-Mozo, The Double Copy Structure of Soft Gravitons, JHEP 03 (2015) 070 [arXiv: 1412.3699] [inSPIRE].

[33] P. Di Vecchia, R. Marotta and M. Mojaza, Soft theorem for the graviton, dilaton and the Kalb-Ramond field in the bosonic string, JHEP 05 (2015) 137 [arXiv: 1502.05258] [INSPIRE].

[34] F. Cachazo, S. He and E.Y. Yuan, New Double Soft Emission Theorems, Phys. Rev. D 92 (2015) 065030 [arXiv:1503.04816] [INSPIRE].

[35] A.E. Lipstein, Soft Theorems from Conformal Field Theory, JHEP 06 (2015) 166 [arXiv: 1504.01364] [INSPIRE].

[36] T. Klose, T. McLoughlin, D. Nandan, J. Plefka and G. Travaglini, Double-Soft Limits of Gluons and Gravitons, JHEP 07 (2015) 135 [arXiv:1504.05558] [INSPIRE].

[37] A. Volovich, C. Wen and M. Zlotnikov, Double Soft Theorems in Gauge and String Theories, JHEP 07 (2015) 095 [arXiv: 1504.05559] [INSPIRE].

[38] M. Bianchi and A.L. Guerrieri, On the soft limit of open string disk amplitudes with massive states, JHEP 09 (2015) 164 [arXiv: 1505.05854] [INSPIRE].

[39] P. Di Vecchia, R. Marotta and M. Mojaza, Double-soft behavior for scalars and gluons from string theory, JHEP 12 (2015) 150 [arXiv: 1507.00938] [INSPIRE].

[40] A.L. Guerrieri, Soft behavior of string amplitudes with external massive states, Nuovo Cim. C 39 (2016) 221 [arXiv:1507.08829] [INSPIRE]. 
[41] S.D. Alston, D.C. Dunbar and W.B. Perkins, n-point amplitudes with a single negative-helicity graviton, Phys. Rev. D 92 (2015) 065024 [arXiv:1507.08882] [INSPIRE].

[42] Y.-t. Huang and C. Wen, Soft theorems from anomalous symmetries, JHEP 12 (2015) 143 [arXiv: 1509.07840] [INSPIRE].

[43] P. Di Vecchia, R. Marotta and M. Mojaza, Soft Theorems from String Theory, Fortsch. Phys. 64 (2016) 389 [arXiv: 1511.04921] [INSPIRE].

[44] M. Bianchi and A.L. Guerrieri, On the soft limit of closed string amplitudes with massive states, Nucl. Phys. B 905 (2016) 188 [arXiv:1512.00803] [INSPIRE].

[45] M. Bianchi and A.L. Guerrieri, On the soft limit of tree-level string amplitudes, arXiv: 1601.03457 [INSPIRE].

[46] J. Rao and B. Feng, Note on Identities Inspired by New Soft Theorems, JHEP 04 (2016) 173 [arXiv: 1604.00650] [INSPIRE].

[47] P. Di Vecchia, R. Marotta and M. Mojaza, Subsubleading soft theorems of gravitons and dilatons in the bosonic string, JHEP 06 (2016) 054 [arXiv:1604.03355] [INSPIRE].

[48] S. He, Z. Liu and J.-B. Wu, Scattering Equations, Twistor-string Formulas and Double-soft Limits in Four Dimensions, JHEP 07 (2016) 060 [arXiv: 1604.02834] [INSPIRE].

[49] F. Cachazo, P. Cha and S. Mizera, Extensions of Theories from Soft Limits, JHEP 06 (2016) 170 [arXiv: 1604.03893] [INSPIRE].

[50] A.P. Saha, Double Soft Theorem for Perturbative Gravity, JHEP 09 (2016) 165 [arXiv: 1607.02700] [INSPIRE].

[51] P. Di Vecchia, R. Marotta and M. Mojaza, Soft behavior of a closed massless state in superstring and universality in the soft behavior of the dilaton, JHEP 12 (2016) 020 [arXiv: 1610.03481] [INSPIRE].

[52] A. Luna, S. Melville, S.G. Naculich and C.D. White, Next-to-soft corrections to high energy scattering in QCD and gravity, JHEP 01 (2017) 052 [arXiv:1611.02172] [INSPIRE].

[53] H. Elvang, C.R.T. Jones and S.G. Naculich, Soft Photon and Graviton Theorems in Effective Field Theory, Phys. Rev. Lett. 118 (2017) 231601 [arXiv:1611.07534] [InSPIRE].

[54] C. Cheung, K. Kampf, J. Novotny, C.-H. Shen and J. Trnka, A Periodic Table of Effective Field Theories, JHEP 02 (2017) 020 [arXiv: 1611.03137] [INSPIRE].

[55] A.P. Saha, Double soft limit of the graviton amplitude from the Cachazo-He-Yuan formalism, Phys. Rev. D 96 (2017) 045002 [arXiv:1702.02350] [INSPIRE].

[56] A. Sen, Soft Theorems in Superstring Theory, JHEP 06 (2017) 113 [arXiv:1702.03934] [INSPIRE].

[57] A. Sen, Subleading Soft Graviton Theorem for Loop Amplitudes, JHEP 11 (2017) 123 [arXiv: 1703.00024] [INSPIRE].

[58] P. Di Vecchia, R. Marotta and M. Mojaza, Double-soft behavior of the dilaton of spontaneously broken conformal invariance, JHEP 09 (2017) 001 [arXiv:1705.06175] [INSPIRE].

[59] A. Laddha and A. Sen, Sub-subleading Soft Graviton Theorem in Generic Theories of Quantum Gravity, JHEP 10 (2017) 065 [arXiv:1706.00759] [INSPIRE].

[60] S. Chakrabarti, S.P. Kashyap, B. Sahoo, A. Sen and M. Verma, Subleading Soft Theorem for Multiple Soft Gravitons, JHEP 12 (2017) 150 [arXiv:1707.06803] [INSPIRE]. 
[61] A. Strominger, On BMS Invariance of Gravitational Scattering, JHEP 07 (2014) 152 [arXiv: 1312.2229] [INSPIRE].

[62] T. He, V. Lysov, P. Mitra and A. Strominger, BMS supertranslations and Weinberg's soft graviton theorem, JHEP 05 (2015) 151 [arXiv:1401.7026] [INSPIRE].

[63] M. Campiglia and A. Laddha, Asymptotic symmetries and subleading soft graviton theorem, Phys. Rev. D 90 (2014) 124028 [arXiv:1408.2228] [INSPIRE].

[64] A. Strominger and A. Zhiboedov, Gravitational Memory, BMS Supertranslations and Soft Theorems, JHEP 01 (2016) 086 [arXiv:1411.5745] [INSPIRE].

[65] M. Campiglia and A. Laddha, New symmetries for the Gravitational S-matrix, JHEP 04 (2015) 076 [arXiv: 1502.02318] [INSPIRE].

[66] S. Pasterski, A. Strominger and A. Zhiboedov, New Gravitational Memories, JHEP 12 (2016) 053 [arXiv : 1502.06120] [InSPIRE].

[67] D. Kapec, V. Lysov, S. Pasterski and A. Strominger, Higher-Dimensional Supertranslations and Weinberg's Soft Graviton Theorem, arXiv:1502.07644 [INSPIRE].

[68] M. Campiglia and A. Laddha, Asymptotic symmetries of QED and Weinberg's soft photon theorem, JHEP 07 (2015) 115 [arXiv:1505.05346] [INSPIRE].

[69] S.G. Avery and B.U.W. Schwab, Burg-Metzner-Sachs symmetry, string theory, and soft theorems, Phys. Rev. D 93 (2016) 026003 [arXiv:1506.05789] [INSPIRE].

[70] M. Campiglia and A. Laddha, Asymptotic symmetries of gravity and soft theorems for massive particles, JHEP 12 (2015) 094 [arXiv: 1509. 01406] [INSPIRE].

[71] M. Campiglia and A. Laddha, Sub-subleading soft gravitons: New symmetries of quantum gravity?, Phys. Lett. B 764 (2017) 218 [arXiv:1605.09094] [INSPIRE].

[72] M. Campiglia and A. Laddha, Subleading soft photons and large gauge transformations, JHEP 11 (2016) 012 [arXiv:1605.09677] [InSPIRE].

[73] M. Campiglia and A. Laddha, Sub-subleading soft gravitons and large diffeomorphisms, JHEP 01 (2017) 036 [arXiv:1608.00685] [INSPIRE].

[74] E. Conde and P. Mao, BMS Supertranslations and Not So Soft Gravitons, JHEP 05 (2017) 060 [arXiv: 1612.08294] [INSPIRE].

[75] T. He, D. Kapec, A.-M. Raclariu and A. Strominger, Loop-Corrected Virasoro Symmetry of 4D Quantum Gravity, JHEP 08 (2017) 050 [arXiv: 1701.00496] [INSPIRE].

[76] M. Asorey, A.P. Balachandran, F. Lizzi and G. Marmo, Equations of Motion as Constraints: Superselection Rules, Ward Identities, JHEP 03 (2017) 136 [arXiv:1612.05886] [InSPIRE].

[77] A. Campoleoni, D. Francia and C. Heissenberg, On higher-spin supertranslations and superrotations, JHEP 05 (2017) 120 [arXiv:1703.01351] [INSPIRE].

[78] A. Strominger, Lectures on the Infrared Structure of Gravity and Gauge Theory, arXiv: 1703.05448 [INSPIRE].

[79] M. Pate, A.-M. Raclariu and A. Strominger, Color Memory: A Yang-Mills Analog of Gravitational Wave Memory, Phys. Rev. Lett. 119 (2017) 261602 [arXiv:1707.08016] [INSPIRE].

[80] A. Laddha and P. Mitra, Asymptotic Symmetries and Subleading Soft Photon Theorem in Effective Field Theories, JHEP 05 (2018) 132 [arXiv:1709.03850] [INSPIRE].

[81] D. Kapec and P. Mitra, A d-Dimensional Stress Tensor for Mink ${ }_{d+2}$ Gravity, JHEP 05 (2018) 186 [arXiv: 1711.04371] [INSPIRE]. 
[82] M. Pate, A.-M. Raclariu and A. Strominger, Gravitational Memory in Higher Dimensions, JHEP 06 (2018) 138 [arXiv: 1712.01204] [INSPIRE].

[83] A. Campoleoni, D. Francia and C. Heissenberg, Asymptotic Charges at Null Infinity in Any Dimension, Universe 4 (2018) 47 [arXiv:1712.09591] [INSPIRE].

[84] A.H. Anupam, A. Kundu and K. Ray, Double soft graviton theorems and Bondi-Metzner-Sachs symmetries, Phys. Rev. D 97 (2018) 106019 [arXiv:1803.03023] [INSPIRE].

[85] A. Laddha and A. Sen, Gravity Waves from Soft Theorem in General Dimensions, JHEP 09 (2018) 105 [arXiv : 1801.07719] [InSPIRE].

[86] W.D. Goldberger and A. Ross, Gravitational radiative corrections from effective field theory, Phys. Rev. D 81 (2010) 124015 [arXiv:0912 .4254] [inSPIRE].

[87] R.A. Porto, A. Ross and I.Z. Rothstein, Spin induced multipole moments for the gravitational wave amplitude from binary inspirals to 2.5 Post-Newtonian order, JCAP 09 (2012) 028 [arXiv: 1203.2962] [INSPIRE].

[88] W.D. Goldberger, A. Ross and I.Z. Rothstein, Black hole mass dynamics and renormalization group evolution, Phys. Rev. D 89 (2014) 124033 [arXiv:1211.6095] [INSPIRE].

[89] F. Bloch and A. Nordsieck, Note on the Radiation Field of the electron, Phys. Rev. 52 (1937) 54 [INSPIRE].

[90] T. Kinoshita, Mass singularities of Feynman amplitudes, J. Math. Phys. 3 (1962) 650 [INSPIRE].

[91] T.D. Lee and M. Nauenberg, Degenerate Systems and Mass Singularities, Phys. Rev. 133 (1964) B1549 [INSPIRE].

[92] P.P. Kulish and L.D. Faddeev, Asymptotic conditions and infrared divergences in quantum electrodynamics, Theor. Math. Phys. 4 (1970) 745 [Teor. Mat. Fiz. 4 (1970) 153] [inSPIRE].

[93] J. Ware, R. Saotome and R. Akhoury, Construction of an asymptotic S matrix for perturbative quantum gravity, JHEP 10 (2013) 159 [arXiv:1308.6285] [INSPIRE].

[94] D. Kapec, M. Perry, A.-M. Raclariu and A. Strominger, Infrared Divergences in QED, Revisited, Phys. Rev. D 96 (2017) 085002 [arXiv:1705.04311] [InSPIRE].

[95] B. Gabai and A. Sever, Large gauge symmetries and asymptotic states in QED, JHEP 12 (2016) 095 [arXiv: 1607.08599] [INSPIRE].

[96] J.D. Jackson, Classical Electrodynamics, John Wiley and Sons, (1999).

[97] P.C. Peters, Relativistic gravitational bremsstrahlung, Phys. Rev. D 1 (1970) 1559 [InSPIRE]. 